1 Hacettepe Journal of Mathematics and Statistics

h Volume 45 (2) (2016), 391-416

\title{
New existence results for positive solutions of boundary value problems for coupled systems of multi-term fractional differential equations
}

\author{
Yuji Liu* †
}

\begin{abstract}
In this article, we establish some new existence results on positive solutions of a boundary value problem of coupled systems of nonlinear multi-term fractional differential equations. Our analysis rely on the well known fixed point theorems. Numerical examples are given to illustrate the main theorems.
\end{abstract}

Keywords: Four-point boundary value problem, multi-term fractional differential system, non-Caratheodory function, fixed-point theorem.

2000 AMS Classification: 34A08, 26A33, 39B99, 45G10, 34B37, 34B15, 34B16

Received: 02.12.2014 Accepted: 18.02.2015 Doi : 10.15672/HJMS.20164512499

\section{Introduction}

Fractional differential systems have many applications in modeling of physical and chemical processes and in engineering and have been of great interest recently. In its turn, mathematical aspects of studies on fractional differential systems were discussed by many authors, see the text book [6, 13] and papers [1, 7, 9, 11, 14, 15, 16, 20, 21, 22, 23].

In [20], the author studied the existence of positive solutions (continuous on [0,1]) of the following $(n-1,1)$-type conjugate boundary value problem for the coupled system of the fractional differential equations

$$
\left\{\begin{array}{l}
D_{0+}^{\alpha} u+\lambda f(t, v)=0,0<t<1, \lambda>0 \\
D_{0+}^{\alpha} v+\lambda g(t, u)=0 \\
u^{(i)}(0)=v^{(i)}(0)=0,0 \leq i \leq n-2 \\
u(1)=v(1)=0
\end{array}\right.
$$

*Department of Mathematics, Guangdong University of Finance and Economics, Guangzhou 510000, P.R.China, Email: liuyuji888@sohu.com

${ }^{\dagger}$ Corresponding Author.

¥Supported by the National Natural Science Foundation of China (No: 11401111), the Natural Science Foundation of Guangdong province (No:S2011010001900) and the Foundation for Highlevel talents in Guangdong Higher Education Project. 
where $\lambda$ is a parameter, $\alpha \in(n-1, n]$ is a real number and $n \geq 3$, and $D_{0+}^{\alpha}$ is the Riemann-Liouville's fractional derivative, and $f, g$ are continuous and semipositone. form

In [9], the author studied the system of fractional boundary value problems of the

$$
\begin{cases}D_{0^{+}}^{\alpha} u(t)+\lambda a(t) f(u(t), v(t))=0, & t \in(0,1), \\ D_{0^{+}}^{\beta} v(t)+\mu b(t) g(u(t), v(t))=0, & t \in(0,1), \\ u^{(i)}(0)=0, i=0,1,2, \cdots, n-2, & D_{0^{+}}^{\gamma} u(1)=0,1<\gamma<n-2, \\ v^{(i)}(0)=0, i=0,1,2, \cdots, n-2, & D_{0^{+}}^{\gamma} v(1)=0,1<\gamma<n-2,\end{cases}
$$

where $D_{0+}$ is the Riemann-Liouville fractional derivative, $n-1<\alpha, \beta<n$ for $n>3$ and $n \in N, a$ and $b$ are continuous on $[0,1], f$ and $g$ continuous functions defined on $R^{2}$. Sufficient conditions for the existence of at least one positive solution (continuous on $[0,1])$ of $\operatorname{BVP}(1.2)$ were obtained.

In known literature, $D_{0^{+}}^{\alpha} u(t)+f(t, u(t))=0$ is known as a single term equation. In certain cases, we find equations containing more than one differential terms. A classical example is the so-called Bagley Torvik equation

$$
A D_{0^{+}}^{2} y(x)+B D_{0^{+}}^{\frac{3}{2}} y(x)+C y(x)=f(x),
$$

where $A, B, C$ are constants and $f$ is a given function. This equation arises from for example the modelling of motion of a rigid plate immersed in a Newtonian fluid. It was originally proposed in [18]. Another example for an application of equations with more than one fractional derivatives is the Basset equation

$$
A D_{0^{+}}^{1} y(x)+b D_{0^{+}}^{n} y(x)+c y(x)=f(x), y(0)=y_{0},
$$

where $0<n<1$. This equation is most frequently, but not exclusively, used with $n=\frac{1}{2}$. It describes the forces that occur when a spherical object sinks in a (relatively dense) incompressible viscous fluid, see [4, 12].

In [17], Su investigated the existence of positive solutions (continuous on $[0,1]$ ) of the following boundary value problem of nonlinear multi-term fractional differential system

$$
\left\{\begin{array}{l}
D_{0+}^{\alpha} u+f\left(t, v(t), D_{0+}^{p} v(t)\right)=0,0<t<1, \\
D_{0+}^{\beta} v+g\left(t, u(t), D_{0+}^{q} u(t)\right)=0,0<t<1, \\
u(0)=0, u(1)=0, \quad v(0)=0, v(1)=0
\end{array}\right.
$$

where $\alpha, \beta \in(1,2), D_{0+}$ is the Riemann-Liouville's fractional derivative, $0<p<\beta-1$, $0<q<\alpha-1, \gamma \eta^{\alpha-1}<1$ and $\gamma \eta^{\beta-1}<1, f, g:[0,1] \times \mathbb{R}_{+} \times \mathbb{R} \rightarrow \mathbb{R}$ are continuous functions.

In [21], authors studied the existence of multiple positive solutions (continuous on $[0,1])$ of the following boundary value problem of N-dimension nonlinear fractional differential system

$$
\left\{\begin{array}{l}
D_{0+}^{\alpha_{1}} u_{1}+f_{1}\left(t, u_{2}(t), D_{0^{+}}^{\mu_{1}} u_{2}(t)\right)=0,0<t<1, \\
\cdots \cdots \cdots \cdots, \\
D_{0+}^{\alpha_{N}-1} u_{N-1}+f_{N-1}\left(t, u_{N}(t), D_{0+}^{\mu_{N}} u_{N}(t)\right)=0,0<t<1, \\
D_{0+}^{\alpha_{N}} u_{N}+f_{N}\left(t, u_{1}(t), D_{0^{+}}^{\mu_{N}} u_{1}(t)\right)=0,0<t<1, \\
u_{1}(0)=\cdots=u_{N}(0)=0, u_{1}(1)=\cdots=u_{N}(1)=0,
\end{array}\right.
$$

where $\alpha_{i} \in(1,2), D_{0+}$ is the Riemann-Liouville's fractional derivative, $0<\mu_{i-1}<\alpha_{i}-1$ with $\mu_{0}=\mu_{N}, f_{i}: ;[0,1] \times \mathbb{R}_{+} \times \mathbb{R} \rightarrow \mathbb{R}(i=1,2, \cdots, N)$ are continuous functions.

In [1], the authors investigated the existence of positive solutions (continuous on $[0,1]$ ) of the following boundary value problem of nonlinear multi-term fractional differential 
system

$$
\left\{\begin{array}{l}
D_{0+}^{\alpha} u+f\left(t, v(t), D_{0+}^{p} v(t)\right)=0,0<t<1 \\
D_{0+}^{\beta} v+g\left(t, u(t), D_{0^{+}}^{q} u(t)\right)=0,0<t<1, \\
u(0)=0, u(1)=\gamma u(\eta) \\
v(0)=0, v(1)=\gamma v(\eta)
\end{array}\right.
$$

where $\alpha, \beta \in(1,2), D_{0+}$ is the Riemann-Liouville's fractional derivative, $0<p \leq \beta-1$, $0<q \leq \alpha-1, \gamma \eta^{\alpha-1}<1$ and $\gamma \eta^{\beta-1}<1, f, g:[0,1] \times \mathbb{R}_{+} \times \mathbb{R} \rightarrow \mathbb{R}$ are continuous functions.

In [24], authors studied the existence of solutions of the following four-point coupled boundary value problem for nonlinear fractional differential equation

$$
\left\{\begin{array}{l}
D_{0+}^{\alpha} u=f\left(t, u(t), D_{0^{+}}^{\alpha-1} u(t), v(t), D_{0^{+}}^{\beta-1} v(t)\right), 0<t<1, \\
D_{0+}^{\beta} v=g\left(t, u(t), D_{0^{+}}^{\alpha-1} u(t), v(t), D_{0^{+}}^{\beta-1} v(t)\right), 0<t<1, \\
I_{0^{+}}^{2-\alpha} u(0)=0, u(1)=a v(\xi), I_{0^{+}}^{2-\beta} v(0)=0, v(1)=b u(\eta),
\end{array}\right.
$$

where $1<\alpha, \beta<2, D_{0^{+}}^{*}$ and $I_{0^{+}}^{*}$ are the standard Riemann-Liouville differentiation and integration, $f, g:[0,1] \times \mathbb{R}^{4} \rightarrow R$ are continuous functions, $a, b \in R, \xi, \eta \in(0,1)$ with $a b \xi^{\beta-1} \eta^{\alpha-1}=1$.

In [8], the existence of positive solutions of the following four-point boundary value problem of multi-term fractional differential system

$$
\left\{\begin{array}{l}
D_{0+}^{\alpha} u=f\left(t, v(t), D_{0^{+}}^{m} v(t)\right), 0<t<1 \\
D_{0+}^{\beta} v=g\left(t, u(t), D_{0^{+}}^{n} u(t)\right), 0<t<1 \\
u(0)=\gamma u(\xi), u(1)=\delta u(\eta), v(0)=\gamma v(\xi), v(1)=\delta v(\eta),
\end{array}\right.
$$

was studied, where $1<\alpha, \beta<2,0<m \leq \beta-1,0<n \leq \alpha-1, \gamma>0, \delta>0,0<\xi<$ $\eta<1, D_{0^{+}}^{*}$ is the standard Riemann-Liouville differentiation, $f, g:[0,1] \times R^{4} \rightarrow R$ are continuous functions and the following assumption (A):

$$
\begin{aligned}
& \max \left\{\delta \eta^{\alpha-1}, \delta \eta^{\alpha-2}\right\}<1, \max \left\{\delta \eta^{\beta-1}, \delta \eta^{\beta-2}\right\}<1, \\
& \max \left\{\gamma \xi^{\alpha-1}, \gamma \xi^{\alpha-2}\right\}<1, \max \left\{\gamma \xi^{\beta-1}, \gamma \xi^{\beta-2}\right\}<1 .
\end{aligned}
$$

In [2], Ahmad and Sivasundaram considered the existence and uniqueness of solutions for the following four-point nonlocal boundary value problem of nonlinear fractional integro-differential equation

$$
\left\{\begin{array}{l}
{ }^{c} D_{0+}^{q} x(t)=f(t, x(t),(\phi x)(t),(\psi x(t)), 0<t<1 \\
x^{\prime}(0)+\alpha x\left(\eta_{1}\right)=0, \quad b x^{\prime}(1)+x\left(\eta_{2}\right)=0
\end{array}\right.
$$

where $1<q \leq 2, a, b \in[0,1], 0<\eta_{1} \leq \eta_{2}<1,{ }^{c} D_{0^{+}}^{q}$ is the Caputo's fractional derivative, $f:[0,1] \times X \times X \times X \rightarrow X$ is continuous, for $\gamma, \delta:[0,1] \times[0,1] \rightarrow[0,+\infty)$ with

$$
(\phi x)(t)=\int_{0}^{t} \gamma(t, s) x(s) d s,(\psi x)(t)=\int_{0}^{1} \delta(t, s) x(s) d s .
$$

We remark that the boundary conditions $x^{\prime}(0)+\alpha x\left(\eta_{1}\right)=0, b x^{\prime}(1)+x\left(\eta_{2}\right)=0$ arise in the study of heat flow problems involving a bar of unit length with two controllers at $t=0$ and $t=1$ adding or removing heat according to the temperatures detected by two sensors at $t=\eta_{1}$ and $t=\eta_{2}$.

We note firstly that the existence of positive solutions of $\operatorname{BVP}(1.7)$ has not been concerned in known papers when the assumption (A) does not hold. Secondly, to guarantee the solvability of $\operatorname{BVP}(1.3)$ and $\operatorname{BVP}(1.5)$ in [1], the assumptions imposed on the 
nonlinearities are as follows:

$$
\begin{aligned}
& |f(t, x, y)| \leq a(t)+\epsilon_{1}|x|^{\rho_{1}}+\epsilon_{2}|y|^{\rho_{2}}, \epsilon_{1}, \epsilon_{2}>0,0<\rho_{1}, \rho_{2}<1, \\
& |g(t, x, y)| \leq b(t)+\delta_{1}|x|^{\sigma_{1}}+\delta_{2}|y|^{\sigma_{2}}, \delta_{1}, \delta_{2}>0,0<\sigma_{1}, \sigma_{2}<1 .
\end{aligned}
$$

While in [17], another assumptions imposed on $f, g$ are as follows:

$$
\begin{aligned}
& |f(t, x, y)| \leq \epsilon_{1}|x|^{\rho_{1}}+\epsilon_{2}|y|^{\rho_{2}}, \epsilon_{1}, \epsilon_{2}>0, \rho_{1}, \rho_{2}>1, \\
& |g(t, x, y)| \leq \delta_{1}|x|^{\sigma_{1}}+\delta_{2}|y|^{\sigma_{2}}, \delta_{1}, \delta_{2}>0, \sigma_{1}, \sigma_{2}>1 .
\end{aligned}
$$

By carefully checking Example 3.1 in [17], one finds that the solution obtained may be the zero solution. This fact makes these papers far from perfect. Thirdly, it is easy to show that the following problem

$$
D_{0^{+}}^{\frac{7}{3}} x(t)=-t^{-\frac{1}{2}}(1-t)^{-\frac{5}{4}}, \lim _{t \rightarrow 0} t^{\frac{2}{3}} x(t)=0, x(1)=0
$$

has a continuous solution on $[0,1]$

$$
x(t)=-\int_{0}^{t} \frac{(t-s)^{\frac{4}{3}}}{\Gamma(7 / 3)} s^{-\frac{1}{2}}(1-s)^{-\frac{5}{4}} d s+t^{\frac{4}{3}} \int_{0}^{1} \frac{(1-s)^{\frac{1}{12}}}{\Gamma(7 / 3)} s^{-\frac{1}{2}} d s,
$$

while $t^{-\frac{1}{2}}(1-t)^{-\frac{5}{4}}$ is not measurable on $(0,1)$. Hence it is interesting to investigate the solvability of mentioned problems with non-Caratheodory functions.

Motivated by above mentioned papers, we discuss the existence of solutions of the following boundary value problem of the multi-term fractional differential system

$$
\left\{\begin{array}{l}
D_{0^{+}}^{\alpha} u(t)+p(t) f\left(t, v(t), D_{0^{+}}^{n} v(t)\right)=0, \quad t \in(0,1), \\
D_{0^{+}}^{\beta} v(t)+q(t) g\left(t, u(t), D_{0^{+}}^{m} u(t)\right)=0, \quad t \in(0,1), \\
\lim _{t \rightarrow 0} t^{2-\alpha} u(t)-a u(\xi)=\int_{0}^{1} \phi_{1}\left(t, v(t), D_{0^{+}}^{n} v(t)\right) d t, \\
u(1)-b u(\eta)=\int_{0}^{1} \psi_{1}\left(t, v(t), D_{0^{+}}^{n} v(t)\right) d t, \\
\lim _{t \rightarrow 0} t^{2-\beta} v(t)-c v(\xi)=\int_{0}^{1} \phi_{2}\left(t, u(t), D_{0^{+}}^{m} u(t)\right) d t, \\
v(1)-d v(\eta)=\int_{0}^{1} \psi_{2}\left(t, u(t), D_{0^{+}}^{m} u(t)\right) d t,
\end{array}\right.
$$

where

(i) $1<\alpha, \beta \leq 2,0<m \leq \alpha-1$ and $0<n \leq \beta-1, D_{0^{+}}^{*}$ is the standard RiemannLiouville differentiation of order $*>0$,

(ii) $0<\xi \leq \eta<1$ and $a, b, c, d \geq 0$,

(iii) $\mathbb{R}$ denote the set of real numbers and $\mathbb{R}_{+}$the set of nonnegative real numbers, $p, q:(0,1) \rightarrow \mathbb{R}_{+}, p$ satisfies that there exist numbers $k_{1}, l_{1}$ such that $k_{1}>-1, \alpha-m+$ $l_{1}>0,2+k_{1}+l_{1}>0$ and $|p(t)|<t^{k_{1}}(1-t)^{l_{1}}$ for $t \in(0,1), q$ satisfies that there exist numbers $k_{2}, l_{2}$ such that $k_{2}>-1, \beta-n+l_{2}>0,2+k_{2}+l_{2}>0$ and $|q(t)|<t^{k_{2}}(1-t)^{l_{2}}$ for $t \in(0,1)$, with $p(t) \not \equiv 0$ and $q(t) \not \equiv 0$ on $(0,1)$,

(iv) $f, \phi_{1}, \psi_{1}:(0,1) \times \mathbb{R}_{+} \times \mathbb{R} \rightarrow \mathbb{R}_{+}$are $(n, \beta)$-Caratheory functions and $g, \phi_{2}, \psi_{2}$ : $(0,1) \times \mathbb{R}_{+} \times \mathbb{R} \rightarrow \mathbb{R}_{+}$is a $(m, \alpha)$-Caratheory functions with $f(t, 0,0) \not \equiv 0$ and $g(t, 0,0) \not \equiv$ 0 on $(0,1)$.

We obtain the results on positive solutions of BVP(1.8) by using Schauder's fixed point theorem in Banach spaces. A pair of functions $(x, y)$ is called a solution of $\mathrm{BVP}(1.8)$ if $x, y \in C^{0}(0,1]$ and $x, y$ satisfy all equations in (1.8). A pair of functions $(x, y)$ is called a positive solution of $\operatorname{BVP}(1.8)$ if $x, y \in C^{0}(0,1]$ are positive on $(0,1]$ and $x, y$ satisfy all equations in (1.8). 
The salient features of the present study are as follows:

(a) the fractional differential equations in (1.8) are multi-term ones and their nonlinearities depend on the lower order fractional derivatives with order greater than $\alpha-1$ and $\beta-1$;

(b) instead of the condition $u(0)=0, v(0)=0$ we consider integral boundary conditions which are more suitable as $D_{0^{+}}^{\alpha} x(t)=0$ with $\alpha \in(1,2)$ implies $x(t)=c t^{\alpha-1}+d t^{\alpha-2}$ and obviously $x$ is not continuous at $t=0$ while $\lim _{t \rightarrow 0^{+}} t^{2-\alpha} x(t)$ exists;

(c) $\mathrm{BVP}(1.8)$ is a generalized form of known ones in references, the positive solutions of $\operatorname{BVP}(1.8)$ obtained are unbounded (discontinuous at $t=0$ ) which are different from those ones (continuous on $[0,1]$ ) in $[1,21,20,9]$;

(d) since $p, q$ may be un-measurable on $(0,1), p(t) f(t, x, y)$ and $q(t) g(t, x, y)$ may be non-Caratheodory functions (see Example 4.1 in which the nonlinearities are

$$
t^{-\frac{1}{10}}(1-t)^{-\frac{21}{20}} f\left(t, v(t), D_{0^{+}}^{\frac{17}{20}} v(t)\right), t^{-\frac{1}{10}}(1-t)^{-\frac{23}{20}} g\left(t, u(t), D_{0^{+}}^{\frac{4}{5}} u(t)\right)
$$

with

$$
\begin{aligned}
& f(t, u, v)=t^{2}+b_{1} t u^{\epsilon_{1}}+a_{1} t v^{\delta_{1}}, a_{1}, b_{1} \geq 0, \epsilon_{1}, \delta_{1}>0, \\
& \left.g(t, u, v)=t^{5}+b_{2} t u^{\sigma_{1}}+a_{2} t v^{\gamma_{1}}, a_{2}, b_{2} \geq 0, \sigma_{1}, \gamma_{1}>0\right) .
\end{aligned}
$$

It is easy to see that both $t^{-\frac{1}{10}}(1-t)^{-\frac{21}{20}}$ and $t^{-\frac{1}{10}}(1-t)^{-\frac{23}{20}}$ are not measurable on $(0,1)$. Our results are new and are well illustrated with an example.

(e) The Green's function $G(t, s)$ for the problem $\left.-D_{0^{+}}^{\alpha} x(t)\right)=0$, $\lim _{t \rightarrow 0^{+}} t^{2-\alpha} x(t)-$ $a x(\xi)=0, x(1)-b x(\eta)=0$ is obtained. We proved that $G(t, s) \geq 0$ under some assumptions which are more weaker than (A) in [8] and actually generalize Lemma 2.2 in ([10] J. Math. Anal. Appl. 305 (2005) 253-276) for problem $-x^{\prime \prime}(t)=0, x(0)-a x(\xi)=$ $x(1)-b x(\eta)=0$. See Lemma 2.9 .

The remainder of this paper is arranged as follows: in Section 2, we present preliminary results; in Section 3, the main result is presented; and two examples are given in Section 4 to illustrate the main result.

\section{Preliminary results}

For the convenience of readers, we present here the necessary definitions from fixed point theory and fractional calculus theory.

2.1. Definition. Let $X$ be a Banach space. An operator $T: X \rightarrow X$ is completely continuous if it is continuous and maps bounded sets into relatively compact sets [3].

2.2. Definition. The Riemann-Liouville fractional integral of order $\alpha>0$ of a function $f:(0,+\infty) \rightarrow R$ is given by $I_{0^{+}}^{\alpha} f(t)=\frac{1}{\Gamma(\alpha)} \int_{0}^{t}(t-s)^{\alpha-1} f(s) d s$, provided that the right-hand side exists [13].

2.3. Definition. The Riemann-Liouville fractional derivative of order $\alpha>0$ of a continuous function $f:(0,+\infty) \rightarrow R$ is given by $D_{0^{+}}^{\alpha} f(t)=\frac{d^{n}}{d t^{n}} \int_{0}^{t} \frac{(t-s)^{n-\alpha-1} f(s)}{\Gamma(n-\alpha)} d s$, where $n-1 \leq \alpha<n$, provided that the right-hand side exists [13].

2.4. Definition. $h:(0,1) \times \mathbb{R} \times \mathbb{R} \rightarrow \mathbb{R}$ is called a $(m, \alpha)$-Carathédory function if it satisfies

(i) $t \rightarrow h\left(t, t^{\alpha-2} x, t^{2+m-\alpha} y\right)$ is measurable on $(0,1)$ for all $(x, y) \in \mathbb{R}^{2}$,

(ii) $(x, y) \rightarrow h\left(t, t^{\alpha-2} x, t^{2+m-\alpha} y\right)$ is continuous for a.e. $t \in(0,1)$,

(iii) for each $r>0$, there exists nonnegative function $\phi_{r} \in L^{1}(0,1)$ such that $|u|,|v| \leq$ $r$ imply $\left|h\left(t, t^{\alpha-2} x, t^{2+m-\alpha} y\right)\right| \leq \phi_{r}(t)$, a.e., $t \in(0,1)$. 
2.5. Lemma. Let $n-1 \leq \alpha<n, u \in C^{0}(0, b) \bigcap L^{1}(0, b)$ with $b>0$. Then

$$
D_{0^{+}}^{\alpha} I_{0^{+}}^{\alpha} u(t)=u(t), \quad I_{0+}^{\alpha} D_{0+}^{\alpha} u(t)=u(t)+C_{1} t^{\alpha-1}+C_{2} t^{\alpha-2}+\cdots+C_{n} t^{\alpha-n},
$$

where $C_{i} \in R, i=1,2, \ldots n[13]$.

Choose

$$
X=\left\{\begin{array}{cc}
x, D_{0^{+}}^{m} x \in C^{0}(0,1] \\
x:(0,1] \rightarrow \mathbb{R} & \text { the following limits exist } \\
& \lim _{t \rightarrow 0} t^{2-\alpha} x(t), \lim _{t \rightarrow 0} t^{2+m-\alpha} D_{0^{+}}^{m} x(t)
\end{array}\right\}
$$

with the norm

$$
\|x\|=\|x\|_{X}=\max \left\{\sup _{t \in(0,1]} t^{2-\alpha}|x(t)|, \sup _{t \in(0,1]} t^{2+m-\alpha}\left|D_{0^{+}}^{m} x(t)\right|\right\}
$$

for $x \in X$. It is easy to show that $X$ is a real Banach space.

Choose

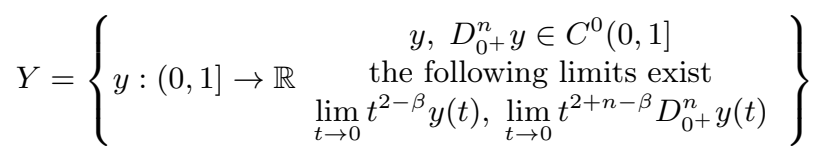

with the norm

$$
\|y\|=\|y\|_{Y}=\max \left\{\sup _{t \in(0,1]} t^{2-\beta}|y(t)|, \sup _{t \in(0,1]} t^{2+n-\beta}\left|D_{0+}^{n} y(t)\right|\right\}
$$

for $y \in Y$. It is easy to show that $Y$ is a real Banach space.

Thus, $(X \times Y,\|\cdot\|)$ is Banach space with the norm defined by

$$
\|(x, y)\|=\max \left\{\|x\|=\|x\|_{X},\|y\|=\|y\|_{Y}\right\} \text { for }(x, y) \in X \times Y .
$$

For ease expression, we denote $F_{m, x}(t)=F\left(t, x(t), D_{0^{+}}^{m} x(t)\right)$ for a function $x:(0,1] \rightarrow$ $\mathbb{R}$, a number $m$ and a function $F:(0,1) \times \mathbb{R}^{2} \rightarrow \mathbb{R}$.

Denote

$$
\begin{aligned}
& \mu_{1}=a \xi^{\alpha-1}, v_{1}=1-a \xi^{\alpha-2}, \omega_{1}=1-b \eta^{\alpha-1}, \lambda_{1}=1-b \eta^{\alpha-2} \\
& \Delta=\mu_{1} \lambda_{1}+v_{1} \omega_{1} \\
& \mu_{2}=c \xi^{\beta-1}, v_{2}=1-c \xi^{\beta-2}, \omega_{2}=1-d \eta^{\beta-1}, \lambda_{2}=1-d \eta^{\beta-2} \\
& \nabla=\mu_{2} \lambda_{2}+v_{2} \omega_{2} .
\end{aligned}
$$

2.6. Lemma. Suppose that $\Delta \neq 0$ and

(B0) $h \in C^{0}(0,1)$ and there exist $k>-1$ and $l \leq 0$ such that $2+l+k>0$ and $|h(t)| \leq t^{k}(1-t)^{l}$ for all $t \in(0,1)$.

Then $x \in X$ is a solution of problem

$$
\left\{\begin{array}{l}
D^{\alpha} x(t)+h(t)=0,0<t<1 \\
\lim _{t \rightarrow 0} t^{2-\alpha} x(t)-a x(\xi)=M \\
x(1)-b x(\eta)=N
\end{array}\right.
$$


if and only if $x \in X$ satisfies

$$
\begin{aligned}
& x(t)=\frac{v_{1} t^{\alpha-1}+\mu_{1} t^{\alpha-2}}{\Delta} N+\frac{\omega_{1} t^{\alpha-2}-\lambda_{1} t^{\alpha-1}}{\Delta} M \\
& -\int_{0}^{t} \frac{(t-s)^{\alpha-1}}{\Gamma(\alpha)} h(s) d s+\frac{v_{1} t^{\alpha-1}+\mu_{1} t^{\alpha-2}}{\Delta} \int_{0}^{1} \frac{(1-s)^{\alpha-1}}{\Gamma(\alpha)} h(s) d s \\
& -\frac{b v_{1} t^{\alpha-1}+b \mu_{1} t^{\alpha-2}}{\Delta} \int_{0}^{\eta} \frac{(\eta-s)^{\alpha-1}}{\Gamma(\alpha)} h(s) d s+\frac{a \lambda_{1} t^{\alpha-1}-a \omega_{1} t^{\alpha-2}}{\Delta} \int_{0}^{\xi} \frac{(\xi-s)^{\alpha-1}}{\Gamma(\alpha)} h(s) d s .
\end{aligned}
$$

Proof. From (B0), we have

$$
\begin{aligned}
& t^{2-\alpha}\left|\int_{0}^{t} \frac{(t-s)^{\alpha-1}}{\Gamma(\alpha)} h(s) d s\right| \leq t^{2-\alpha} \int_{0}^{t} \frac{(t-s)^{\alpha-1}}{\Gamma(\alpha)} s^{k}(1-s)^{l} d s \\
& \leq t^{2-\alpha} \int_{0}^{t} \frac{(t-s)^{\alpha+l-1}}{\Gamma(\alpha)} s^{k} d s=t^{\alpha+l+k} \int 0^{1} \frac{(1-w)^{\alpha+l-1}}{\Gamma(\alpha)} w^{k} d w \\
& =t^{2+l+k} \frac{\mathbf{B}(\alpha+l, k+1)}{\Gamma(\alpha)} \rightarrow 0 \text { as } t \rightarrow 0 .
\end{aligned}
$$

Suppose that $x \in X$ is a solution of (2.2). Lemma 2.5 implies that there exist $c_{i}(i=1,2)$ such that

$$
x(t)=-\int_{0}^{t} \frac{(t-s)^{\alpha-1}}{\Gamma(\alpha)} h(s) d s+c_{1} t^{\alpha-1}+c_{2} t^{\alpha-2} .
$$

One sees from the boundary conditions in (2.2) that

$$
\begin{aligned}
& \mu_{1} c_{1}-v_{1} c_{2}=-M+a \int_{0}^{\xi} \frac{(\xi-s)^{\alpha-1}}{\Gamma(\alpha)} h(s) d s \\
& \omega_{1} c_{1}+\lambda_{1} c_{2}=N+\int_{0}^{1} \frac{(1-s)^{\alpha-1}}{\Gamma(\alpha)} h(s) d s-b \int_{0}^{\eta} \frac{(\eta-s)^{\alpha-1}}{\Gamma(\alpha)} h(s) d s .
\end{aligned}
$$

It follows that

$$
\begin{aligned}
& c_{1}=\frac{1}{\Delta}\left[v_{1} N-\lambda_{1} M+v_{1} \int_{0}^{1} \frac{(1-s)^{\alpha-1}}{\Gamma(\alpha)} h(s) d s\right. \\
& \left.-b v_{1} \int_{0}^{\eta} \frac{(\eta-s)^{\alpha-1}}{\Gamma(\alpha)} h(s) d s+a \lambda_{1} \int_{0}^{\xi} \frac{(\xi-s)^{\alpha-1}}{\Gamma(\alpha)} h(s) d s\right] \\
& c_{2}=\frac{1}{\Delta}\left[\mu_{1} N+\omega_{1} M+\mu_{1} \int_{0}^{1} \frac{(1-s)^{\alpha-1}}{\Gamma(\alpha)} h(s) d s\right. \\
& \left.-b \mu_{1} \int_{0}^{\eta} \frac{(\eta-s)^{\alpha-1}}{\Gamma(\alpha)} h(s) d s-a \omega_{1} \int_{0}^{\xi} \frac{(\xi-s)^{\alpha-1}}{\Gamma(\alpha)} h(s) d s\right] .
\end{aligned}
$$

Substitute $c_{1}, c_{2}$ into (2.4), we get (2.3).

On the other hand, if $x \in X$ satisfies (2.3), we can show that $x \in X$ is a solution of $\operatorname{BVP}(2.2)$. The proof is completed. 
2.7. Lemma. Suppose that $\nabla \neq 0$ and (B0) holds. Then $y \in Y$ is a solution of problem

$$
\left\{\begin{array}{l}
D^{\beta} y(t)+h(t)=0,0<t<1, \\
\lim _{t \rightarrow 0} t^{2-\beta} y(t)-c y(\xi)=M, \\
y(1)-d y(\eta)=N
\end{array}\right.
$$

if and only if $y \in Y$ satisfies

$$
\begin{aligned}
& y(t)=\frac{v_{2} t^{\beta-1}+\mu_{2} t^{\beta-2}}{\nabla} N+\frac{\omega_{2} t^{\beta-2}-\lambda_{2} t^{\beta-1}}{\nabla} M \\
& -\int_{0}^{t} \frac{(t-s)^{\beta-1}}{\Gamma(\beta)} h(s) d s+\frac{v_{2} t^{\beta-1}+\mu_{2} t^{\beta-2}}{\nabla} \int_{0}^{1} \frac{(1-s)^{\beta-1}}{\Gamma(\beta)} h(s) d s \\
& -\frac{d \lambda_{2} t^{\beta-1}+d \mu_{2} t^{\beta-2}}{\nabla} \int_{0}^{\eta} \frac{(\eta-s)^{\beta-1}}{\Gamma(\beta)} h(s) d s+\frac{c \lambda_{2} t^{\beta-1}-c \omega_{2} t^{\beta-2}}{\nabla} \int_{0}^{\xi} \frac{(\xi-s)^{\beta-1}}{\Gamma(\beta)} h(s) d s .
\end{aligned}
$$

Proof. The proof is similar to that of Lemma 2.6 and is omitted.

Define the operator $T$ on $X \times Y$ by $T(x, y)(t)=\left(\left(T_{1} y\right)(t),\left(T_{2} x\right)(t)\right)$ with

$$
\begin{aligned}
& \left(T_{1} y\right)(t)=\frac{v_{1} t^{\alpha-1}+\mu_{1} t^{\alpha-2}}{\Delta} \int_{0}^{1} \psi_{1_{n, y}}(s) d s+\frac{\omega_{1} t^{\alpha-2}-\lambda_{1} t^{\alpha-1}}{\Delta} \int_{0}^{1} \phi_{1_{n, y}}(s) d s \\
& -\int_{0}^{t} \frac{(t-s)^{\alpha-1}}{\Gamma(\alpha)} p(s) f_{n, y}(s) d s+\frac{v_{1} t^{\alpha-1}+\mu_{1} t^{\alpha-2}}{\Delta} \int_{0}^{1} \frac{(1-s)^{\alpha-1}}{\Gamma(\alpha)} p(s) f_{n, y}(s) d s \\
& -\frac{b v_{1} t^{\alpha-1}+b \mu_{1} t^{\alpha-2}}{\Delta} \int_{0}^{\eta} \frac{(\eta-s)^{\alpha-1}}{\Gamma(\alpha)} p(s) f_{n, x}(s) d s \\
& +\frac{a \lambda_{1} t^{\alpha-1}-a \omega_{1} t^{\alpha-2}}{\Delta} \int_{0}^{\xi} \frac{(\xi-s)^{\alpha-1}}{\Gamma(\alpha)} p(s) f_{n, y}(s) d s
\end{aligned}
$$

and

$$
\begin{aligned}
& \left(T_{2} x\right)(t)=\frac{v_{2} t^{\beta-1}+\mu_{2} t^{\beta-2}}{\nabla} \int_{0}^{1} \psi_{2}{ }_{m, x}(s) d s+\frac{\omega_{2} t^{\beta-2}-\lambda_{2} t^{\beta-1}}{\nabla} \int_{0}^{1} \phi_{2}{ }_{m, x}(s) d s \\
& -\int_{0}^{t} \frac{(t-s)^{\beta-1}}{\Gamma(\beta)} q(s) g_{m, x}(s) d s+\frac{v_{2} t^{\beta-1}+\mu_{2} t^{\beta-2}}{\nabla} \int_{0}^{1} \frac{(1-s)^{\beta-1}}{\Gamma(\beta)} q(s) g_{m, x}(s) d s \\
& -\frac{d v_{2} t^{\beta-1}+d \mu_{2} t^{\beta-2}}{\nabla} \int_{0}^{\eta} \frac{(\eta-s)^{\beta-1}}{\Gamma(\beta)} q(s) g_{m, x}(s) d s \\
& +\frac{c \lambda_{2} t^{\beta-1}-c \omega_{2} t^{\beta-2}}{\nabla} \int_{0}^{\xi} \frac{(\xi-s)^{\beta-1}}{\Gamma(\beta)} q(s) g_{m, x}(s) d s
\end{aligned}
$$

for $(x, y) \in X \times Y$.

By Lemmas 2.6 and 2.7, we have that $(x, y) \in X \times Y$ is a solution of $\operatorname{BVP}(1.8)$ if and only if $(x, y) \in X \times Y$ is a fixed point of $T$.

2.8. Lemma. Suppose that (i)-(iv) defined in Section 1 hold, $\Delta \neq 0$ and $\nabla \neq 0$. Then $T: X \times Y \rightarrow X \times Y$ is completely continuous. 
Proof. If $0<m \leq \alpha-1$ and $0<n \leq \beta-1$, we take

$$
\begin{aligned}
& \left.t^{2-\alpha}\left(T_{1} y\right)(t)\right|_{t=0}=\lim _{t \rightarrow 0} t^{2-\alpha}\left(T_{1} y\right)(t), \\
& \left.t^{2+m-\alpha} D_{0^{+}}^{m}\left(T_{1} y\right)(t)\right|_{t=0}=\lim _{t \rightarrow 0} t^{2+m-\alpha} D_{0^{+}}^{m}\left(T_{1} y\right)(t), \\
& \left.t^{2-\beta}\left(T_{2} x\right)(t)\right|_{t=0}=\lim _{t \rightarrow 0} t^{2-\beta}\left(T_{2} x\right)(t), \\
& \left.t^{2+n-\beta} D_{0^{+}}^{n}\left(T_{2} x\right)(t)\right|_{t=0}=\lim _{t \rightarrow 0} t^{2+n-\beta} D_{0^{+}}^{n}\left(T_{2} x\right)(t),
\end{aligned}
$$

then $t^{2-\alpha}\left(T_{1} y\right)(t), t^{2+m-\alpha} D_{0^{+}}^{m}\left(T_{1} y\right)(t)$ and $t^{2-\beta}\left(T_{2} x\right)(t), t^{2+n-\beta} D_{0^{+}}^{n}\left(T_{2} x\right)(t)$ are continuous on $[0,1]$ for each $(x, y) \in X \times Y$. It is easy to show that $T$ is completely continuous, we refer similar proofs to [1]. The proof is complete.

Now, we rewrite

$$
\begin{aligned}
& (T(x, y))(t)=\left(\left(T_{1} y\right)(t),\left(T_{2} x\right)(t)\right) \\
& =\left(\frac{v_{1} t^{\alpha-1}+\mu_{1} t^{\alpha-2}}{\Delta} \int_{0}^{1} \psi_{1_{n, y}}(s) d s+\frac{\omega_{1} t^{\alpha-2}-\lambda_{1} t^{\alpha-1}}{\Delta} \int_{0}^{1} \phi_{1_{n, y}}(s) d s\right. \\
& +\int_{0}^{1} G(t, s) p(s) f_{n, y}(s) d s, \\
& \left.\frac{v_{2} t^{\beta-1}+\mu_{2} t^{\beta-2}}{\nabla} \int_{0}^{1} \psi_{2_{m, x}}(s) d s+\frac{\omega_{2} t^{\beta-2}-\lambda_{2} t^{\beta-1}}{\nabla} \int_{0}^{1} \phi_{2_{m, x}}(s) d s+\int_{0}^{1} H(t, s) g_{m, x}(s) d s\right) .
\end{aligned}
$$

Here

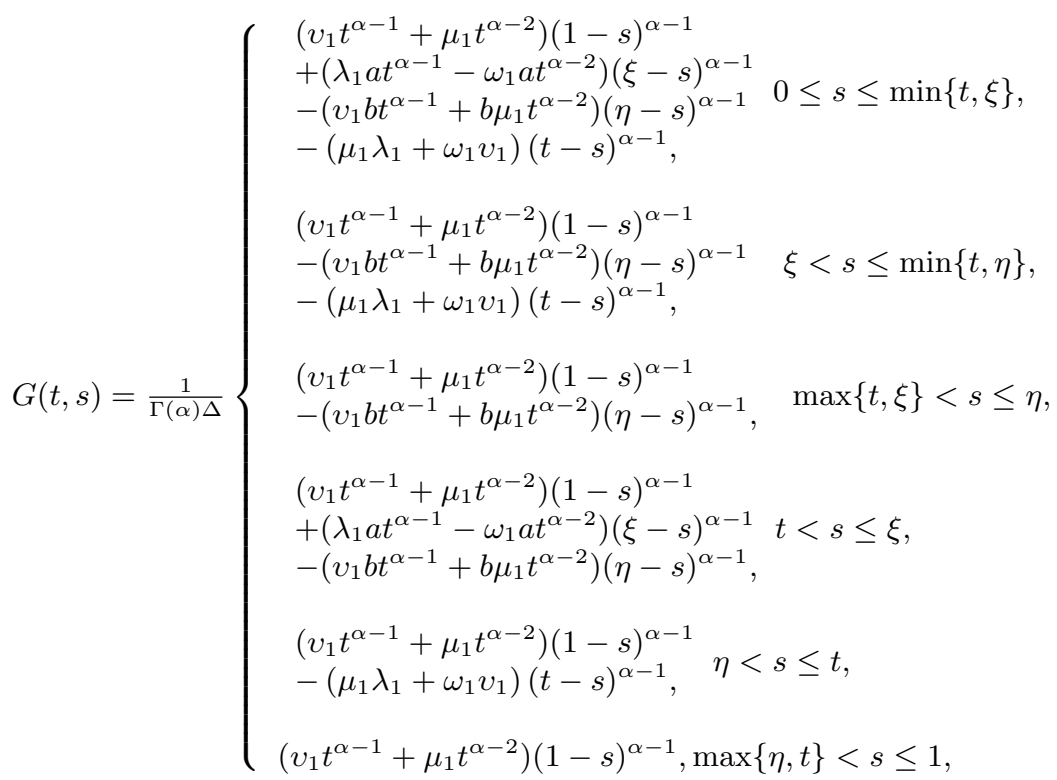


and

$$
H(t, s)=\frac{1}{\Gamma(\beta) \nabla}\left\{\begin{array}{l}
\left(v_{2} t^{\beta-1}+\mu_{2} t^{\beta-2}\right)(1-s)^{\beta-1} \\
+\left(\lambda_{2} c t^{\beta-1}-\omega_{2} c t^{\beta-2}\right)(\xi-s)^{\beta-1} \quad 0 \leq s \leq \min \{t, \xi\}, \\
-\left(\mu_{2} \lambda_{2}+\omega_{2} v_{2}\right)(t-s)^{\beta-1}, \\
\left(v_{2} t^{\beta-1}+\mu_{2} t^{\beta-2}\right)(1-s)^{\beta-1} \\
-\left(v_{2} d t^{\beta-1}+d \mu_{2} t^{\beta-2}\right)(\eta-s)^{\beta-1} \quad \xi<s \leq \min \{t, \eta\}, \\
-\left(\mu_{2} \lambda_{2}+\omega_{2} v_{2}\right)(t-s)^{\beta-1}, \\
\left(v_{2} t^{\beta-1}+\mu_{2} t^{\beta-2}\right)(1-s)^{\beta-1} \\
-\left(v_{2} d t^{\beta-1}+d \mu_{2} t^{\beta-2}\right)(\eta-s)^{\beta-1}, \quad \max \{t, \xi\}<s \leq \eta, \\
\left(v_{2} t^{\beta-1}+\mu_{2} t^{\beta-2}\right)(1-s)^{\beta-1} \\
+\left(\lambda_{2} c t^{\beta-1}-\omega_{2} c t^{\beta-2}\right)(\xi-s)^{\beta-1} \quad t<s \leq \xi, \\
-\left(v_{2} d t^{\beta-1}+d \mu_{2} t^{\beta-2}\right)(\eta-s)^{\beta-1}, \\
\left(v_{2} t^{\beta-1}+\mu_{2} t^{\beta-2}\right)(1-s)^{\beta-1} \\
-\left(\mu_{2} \lambda_{2}+\omega_{2} v_{2}\right)(t-s)^{\beta-1}, \quad \eta<s \leq t, \\
\left(v_{2} t^{\beta-1}+\mu_{2} t^{\beta-2}\right)(1-s)^{\beta-1}, \max \{\eta, t\}<s \leq 1,
\end{array}\right.
$$

2.9. Lemma. Suppose that

$$
\begin{aligned}
& \Delta>0, \quad 0 \leq a<\frac{1}{\xi^{\alpha-2}(1-\xi)}, \quad 0 \leq b<\frac{1}{\eta^{\alpha-1}}, \\
& \nabla>0, \quad 0 \leq c<\frac{1}{\xi^{\beta-2}(1-\xi)}, \quad 0 \leq d<\frac{1}{\eta^{\beta-1}} .
\end{aligned}
$$

Then

$$
G(t, s) \geq 0 \text { for all } t, s \in(0,1), H(t, s) \geq 0 \text { for all } t, s \in(0,1) \text {. }
$$

Proof. By the definitions of $G$, we consider six cases:

Case 1. $0 \leq s \leq \min \{t, \xi\}$. Firstly, from $b \eta^{\alpha-1}<1$ and $0 \leq a \leq \frac{1}{\xi^{\alpha-2}(1-\xi)}$, we have

$$
\begin{aligned}
& \omega_{1} t^{\alpha-1}-\lambda_{1} t^{\alpha-2}=t^{\alpha-2}\left[t-1+b \eta^{\alpha-2}[\eta-t]\right. \\
& \left\{\begin{array}{l}
\leq 0, \quad \eta \leq t, \\
=t^{\alpha-2}\left[t-1+b \eta^{\alpha-1} \frac{\eta-t}{\eta}\right]<t^{\alpha-2}\left[t-1+\frac{\eta-t}{\eta}\right]=t-1+1-\frac{t}{\eta} \leq 0, \eta>t,
\end{array}\right. \\
& \nu_{1} t^{\alpha-1}+b \mu_{1} t^{\alpha-2}=t^{\alpha-2}\left[t-a \xi^{\alpha-2} t+a \xi^{\alpha-1}\right] \\
& \left\{\begin{array}{l}
\geq 0, \quad a \xi^{\alpha-2} \leq 1, \\
\geq 1-a \xi^{\alpha-2}+a \xi^{\alpha-1}>0, a \xi^{\alpha-2}>1 .
\end{array}\right.
\end{aligned}
$$


It is easy to show that $(t-s)^{\alpha-1} \leq t^{\alpha-1}(1-s)^{\alpha-1}$ for all $0 \leq s \leq t$. Then

$$
\begin{aligned}
& -\triangle_{1}(t-s)^{\alpha-1}+\left(\nu_{1} t^{\alpha-1}+\mu_{1} t^{\alpha-2}\right)(1-s)^{\alpha-1}+\left(a \omega_{1} t^{\alpha-1}-a \lambda_{1} t^{\alpha-2}\right)(\xi-s)^{\alpha-1} \\
& -\left(b \nu_{1} t^{\alpha-1}+b \mu_{1} t^{\alpha-2}\right)(\eta-s)^{\alpha-1} \\
& \geq\left[-\triangle_{1} t^{\alpha-1}+\left(\nu_{1} t^{\alpha-1}+\mu_{1} t^{\alpha-2}\right)+\left(a \omega_{1} t^{\alpha-1}-a \lambda_{1} t^{\alpha-2}\right) \xi^{\alpha-1}\right. \\
& \left.-\left(b \nu_{1} t^{\alpha-1}+b \mu_{1} t^{\alpha-2}\right) \eta^{\alpha-1}\right](1-s)^{\alpha-1} \\
& =\left[-a \xi^{\alpha-1}\left(1-b \eta^{\alpha-2}\right) t^{\alpha-1}-\left(1-a \xi^{\alpha-2}\right)\left(1-b \eta^{\alpha-1}\right) t^{\alpha-1}\right. \\
& +\left(\left(1-a \xi^{\alpha-2}\right) t^{\alpha-1}+a \xi^{\alpha-1} t^{\alpha-2}\right)+\left(a\left(1-b \eta^{\alpha-2}\right) t^{\alpha-1}-a\left(1-b \eta^{\alpha-1}\right) t^{\alpha-2}\right) \xi^{\alpha-1} \\
& \left.-\left(b\left(1-a \xi^{\alpha-2}\right) t^{\alpha-1}+a b \xi^{\alpha-1} t^{\alpha-2}\right) \eta^{\alpha-1}\right](1-s)^{\alpha-1}=0 .
\end{aligned}
$$

Case 2. $\max \{t, \eta\}<s \leq 1$. We note that $0 \leq a \leq \frac{1}{\xi^{\alpha-2}(1-\xi)}$. Then

$$
\begin{aligned}
& \nu_{1} t^{\alpha-1}+\mu_{1} t^{\alpha-2}=t^{\alpha-1}-a \xi^{\alpha-2} t^{\alpha-1}+a \xi^{\alpha-1} t^{\alpha-2}=t^{\alpha-2}\left[\left(1-a \xi^{\alpha-2}\right) t+a \xi^{\alpha-1}\right] \\
& \left\{\begin{array}{l}
=t^{\alpha-1}+a \xi^{\alpha-2} t^{\alpha-2}(\xi-t) \geq 0, \xi \geq t \\
\geq 0, \xi<t, a \xi^{\alpha-2} \leq 1 \\
\geq t^{\alpha-2}\left[1-a \xi^{\alpha-2}+a \xi^{\alpha-1}\right] \geq 0, \xi<t, a \xi^{\alpha-2}>1
\end{array}\right.
\end{aligned}
$$

Case 3. $\eta<s \leq t$. From $(t-s)^{\alpha-1} \leq t^{\alpha-1}(1-s)^{\alpha-1}$, we have

$$
\begin{aligned}
& -\triangle_{1}(t-s)^{\alpha-1}+\left(\nu_{1} t^{\alpha-1}+\mu_{1} t^{\alpha-2}\right)(1-s)^{\alpha-1} \\
& =-\triangle_{1}(t-s)^{\alpha-1}+\left(\nu_{1} t^{\alpha-1}+\mu_{1} t^{\alpha-2}\right)(1-s)^{\alpha-1} \\
& +\left(a \omega_{1} t^{\alpha-1}-a \lambda_{1} t^{\alpha-2}\right)(\xi-s)^{\alpha-1}-\left(b \nu_{1} t^{\alpha-1}+b \mu_{1} t^{\alpha-2}\right)(\eta-s)^{\alpha-1} \\
& -\left(a \omega_{1} t^{\alpha-1}-a \lambda_{1} t^{\alpha-2}\right)(\xi-s)^{\alpha-1}+\left(b \nu_{1} t^{\alpha-1}+b \mu_{1} t^{\alpha-2}\right)(\eta-s)^{\alpha-1} \\
& \geq-\triangle_{1}(t-s)^{\alpha-1}+\left(\nu_{1} t^{\alpha-1}+\mu_{1} t^{\alpha-2}\right)(1-s)^{\alpha-1} \\
& +\left(a \omega_{1} t^{\alpha-1}-a \lambda_{1} t^{\alpha-2}\right)(\xi-s)^{\alpha-1}-\left(b \nu_{1} t^{\alpha-1}+b \mu_{1} t^{\alpha-2}\right)(\eta-s)^{\alpha-1} \geq 0 .
\end{aligned}
$$

Case 4. $\xi<s \leq t$. We have

$$
\begin{aligned}
& -\triangle_{1}(t-s)^{\alpha-1}+\left(\nu_{1} t^{\alpha-1}+\mu_{1} t^{\alpha-2}\right)(1-s)^{\alpha-1} \\
& \quad-\left(b \nu_{1} t^{\alpha-1}+b \mu_{1} t^{\alpha-2}\right)(\eta-s)^{\alpha-1} \\
& =-\triangle_{1}(t-s)^{\alpha-1}+\left(\nu_{1} t^{\alpha-1}+\mu_{1} t^{\alpha-2}\right)(1-s)^{\alpha-1} \\
& +\left(a \omega_{1} t^{\alpha-1}-a \lambda_{1} t^{\alpha-2}\right)(\xi-s)^{\alpha-1}-\left(b \nu_{1} t^{\alpha-1}+b \mu_{1} t^{\alpha-2}\right)(\eta-s)^{\alpha-1} \\
& +\triangle_{1}(t-s)^{\alpha-1}+\left(b \nu_{1} t^{\alpha-1}+b \mu_{1} t^{\alpha-2}\right)(\eta-s)^{\alpha-1} \\
& \geq-\triangle_{1}(t-s)^{\alpha-1}+\left(\nu_{1} t^{\alpha-1}+\mu_{1} t^{\alpha-2}\right)(1-s)^{\alpha-1} \\
& +\left(a \omega_{1} t^{\alpha-1}-a \lambda_{1} t^{\alpha-2}\right)(\xi-s)^{\alpha-1}-\left(b \nu_{1} t^{\alpha-1}+b \mu_{1} t^{\alpha-2}\right)(\eta-s)^{\alpha-1} \geq 0 .
\end{aligned}
$$


Case 5. $t<s \leq \xi$. We have

$$
\begin{aligned}
& \left(\nu_{1} t^{\alpha-1}+\mu_{1} t^{\alpha-2}\right)(1-s)^{\alpha-1}+\left(a \omega_{1} t^{\alpha-1}-a \lambda_{1} t^{\alpha-2}\right)(\xi-s)^{\alpha-1} \\
& -\left(b \nu_{1} t^{\alpha-1}+b \mu_{1} t^{\alpha-2}\right)(\eta-s)^{\alpha-1} \\
& =-\triangle_{1}(t-s)^{\alpha-1}+\left(\nu_{1} t^{\alpha-1}+\mu_{1} t^{\alpha-2}\right)(1-s)^{\alpha-1} \\
& +\left(a \omega_{1} t^{\alpha-1}-a \lambda_{1} t^{\alpha-2}\right)(\xi-s)^{\alpha-1} \\
& -\left(b \nu_{1} t^{\alpha-1}+b \mu_{1} t^{\alpha-2}\right)(\eta-s)^{\alpha-1}+\triangle_{1}(t-s)^{\alpha-1} \\
& \geq-\triangle_{1}(t-s)^{\alpha-1}+\left(\nu_{1} t^{\alpha-1}+\mu_{1} t^{\alpha-2}\right)(1-s)^{\alpha-1} \\
& +\left(a \omega_{1} t^{\alpha-1}-a \lambda_{1} t^{\alpha-2}\right)(\xi-s)^{\alpha-1} \\
& -\left(b \nu_{1} t^{\alpha-1}+b \mu_{1} t^{\alpha-2}\right)(\eta-s)^{\alpha-1} \geq 0 .
\end{aligned}
$$

Case 6. $\max \{t, \xi\}<s \leq \eta$. We have

$$
\begin{aligned}
& \left(\nu_{1} t^{\alpha-1}+\mu_{1} t^{\alpha-2}\right)(1-s)^{\alpha-1}-\left(b \nu_{1} t^{\alpha-1}+b \mu_{1} t^{\alpha-2}\right)(\eta-s)^{\alpha-1} \\
& =-\triangle_{1}(t-s)^{\alpha-1}+\left(\nu_{1} t^{\alpha-1}+\mu_{1} t^{\alpha-2}\right)(1-s)^{\alpha-1} \\
& +\left(a \omega_{1} t^{\alpha-1}-a \lambda_{1} t^{\alpha-2}\right)(\xi-s)^{\alpha-1} \\
& -\left(b \nu_{1} t^{\alpha-1}+b \mu_{1} t^{\alpha-2}\right)(\eta-s)^{\alpha-1}+\triangle_{1}(t-s)^{\alpha-1} \\
& -\left(a \omega_{1} t^{\alpha-1}-a \lambda_{1} t^{\alpha-2}\right)(\xi-s)^{\alpha-1} \\
& \geq-\triangle_{1}(t-s)^{\alpha-1}+\left(\nu_{1} t^{\alpha-1}+\mu_{1} t^{\alpha-2}\right)(1-s)^{\alpha-1} \\
& +\left(a \omega_{1} t^{\alpha-1}-a \lambda_{1} t^{\alpha-2}\right)(\xi-s)^{\alpha-1} \\
& -\left(b \nu_{1} t^{\alpha-1}+b \mu_{1} t^{\alpha-2}\right)(\eta-s)^{\alpha-1} \geq 0
\end{aligned}
$$

We know by the definition of $G$ that $G(t, s) \geq 0$ for all $t, s \in(0,1)$. Similarly we can prove that $H(t, s) \geq 0$ for all $t, s \in(0,1)$ The proof is completed.

\section{Main results}

In this section, we prove existence result on solutions of $\operatorname{BVP}(1.8)$. Let $\mu_{i}, v_{i}, \omega_{i}, \lambda_{i}$ $(i=1,2)$ and $\Delta, \nabla$ be defined by (2.1). For $\Phi \in L^{1}(0,1)$, denote $\|\Phi\|_{1}=\int_{0}^{1}|\Phi(s)| d s$. The following assumption will be used in the main theorem.

(B1) there exist nonnegative constants $b_{i}, a_{i}(i=1,2), B_{i}, A_{i}, C_{i}, D_{i}(i=1,2)$ and $\epsilon_{1}, \delta_{i}, \gamma_{i}, \sigma_{i}(i=1,2), \Phi_{i}, \Psi_{i}, \Phi_{i 0}, \Psi_{i 0} \in L^{1}(0,1)(i=1,2)$ and bounded functions $\Phi, \Psi$ 
such that

$$
\begin{aligned}
& \left|f\left(t, \frac{u}{t^{2-\beta}}, \frac{v}{t^{2+n-\beta}}\right)-\Phi(t)\right| \leq b_{1}|u|^{\epsilon_{1}}+a_{1}|v|^{\delta_{1}}, t \in(0,1), u, v \in \mathbb{R}, \\
& \left|g\left(t, \frac{u}{t^{2-\alpha}}, \frac{v}{t^{2+m-\alpha}}\right)-\Psi(t)\right| \leq b_{2}|u|^{\sigma_{1}}+a_{2}|v|^{\gamma_{1}}, t \in(0,1), u, v \in \mathbb{R}, \\
& \left|\phi_{1}\left(t, \frac{u}{t^{2-\beta}}, \frac{v}{t^{2+n-\beta}}\right)-\Phi_{10}(t)\right| \leq \Phi_{1}(t)\left[B_{1}|u|^{\epsilon_{1}}+A_{1}|v|^{\delta_{1}}, t \in(0,1), u, v \in \mathbb{R},\right. \\
& \left|\psi_{1}\left(t, \frac{u}{t^{2-\beta}}, \frac{v}{t^{2+n-\beta}}\right)-\Psi_{10}(t)\right| \leq \Psi_{1}(t)\left[C_{1}|u|^{\epsilon_{1}}+D_{1}|v|^{\delta_{1}}\right], t \in(0,1), u, v \in \mathbb{R}, \\
& \left|\phi_{2}\left(t, \frac{u}{t^{2-\alpha}}, \frac{v}{t^{2+m-\alpha}}\right)-\Phi_{20}(t)\right| \leq \Phi_{2}(t)\left[B_{2}|u|^{\sigma_{1}}+A_{2}|v|^{\gamma_{1}}\right], t \in(0,1), u, v \in \mathbb{R}, \\
& \left|\psi_{2}\left(t, \frac{u}{t^{2-\alpha}}, \frac{v}{t^{2+m-\alpha}}\right)-\Psi_{20}(t)\right| \leq \Psi_{2}(t)\left[C_{2}|u|^{\sigma_{1}}+D_{2}|v|^{\gamma_{1}}\right], t \in(0,1), u, v \in \mathbb{R} .
\end{aligned}
$$

For ease expression, denote

$$
\begin{aligned}
& \bar{\Phi}(t)=\frac{\left(1-a \xi^{\alpha-2}\right) t^{\alpha-1}+a \xi^{\alpha-1} t^{\alpha-2}}{\Delta} \int_{0}^{1} \Psi_{10}(s) d s \\
& +\frac{\left(1-b \eta^{\alpha-1}\right) t^{\alpha-2}-\left(1-b \eta^{\alpha-2}\right) t^{\alpha-1}}{\Delta} \int_{0}^{1} \Phi_{10}(s) d s \\
& -\int_{0}^{t} \frac{(t-s)^{\alpha-1}}{\Gamma(\alpha)} p(s) \Phi(s) d s+\frac{\left(1-a \xi^{\alpha-2}\right) t^{\alpha-1}+a \xi^{\alpha-1} t^{\alpha-2}}{\Delta} \int_{0}^{1} \frac{(1-s)^{\alpha-1}}{\Gamma(\alpha)} p(s) \Phi(s) d s \\
& -\frac{b\left(1-a \xi^{\alpha-2}\right) t^{\alpha-1}+a b \xi^{\alpha-1} t^{\alpha-2}}{\Delta} \int_{0}^{\eta} \frac{(\eta-s)^{\alpha-1}}{\Gamma(\alpha)} p(s) \Phi(s) d s \\
& \left.+\frac{a\left(1-b \eta^{\alpha-2}\right) t^{\alpha-1}-a\left(1-b \eta^{\alpha-1}\right) t^{\alpha-2}}{\Delta} \int_{0}^{\xi} \frac{(\xi-s)^{\alpha-1}}{\Gamma(\alpha)} p(s) \Phi(s)\right) d s \\
& \bar{\Psi}(t)=\frac{\left(1-c \xi^{\beta-2}\right) t^{\beta-1}+c \xi^{\beta-1} t^{\beta-2}}{\nabla} \int_{0}^{1} \Psi_{20}(s) d s \\
& +\frac{\left(1-d \eta^{\beta-1}\right) t^{\beta-2}-\left(1-d \eta^{\beta-2}\right) t^{\beta-1}}{\nabla} \int_{0}^{1} \Phi_{20}(s) d s \\
& -\int_{0}^{t} \frac{(t-s)^{\beta-1}}{\Gamma(\beta)} q(s) \Psi(s) d s+\frac{\left(1-c \xi^{\beta-2}\right) t^{\beta-1}+c \xi^{\beta-1} t^{\beta-2}}{\nabla} \int_{0}^{1} \frac{(1-s)^{\beta-1}}{\Gamma(\beta)} q(s) \Psi(s) d s \\
& -\frac{d\left(1-c \xi^{\beta-2}\right) t^{\beta-1}+c d \xi^{\beta-1} t^{\beta-2}}{\nabla} \int_{0}^{\eta} \frac{(\eta-s)^{\beta-1}}{\Gamma(\beta)} q(s) \Psi(s) d s \\
& +\frac{c\left(1-d \eta^{\beta-2}\right) t^{\beta-1}-c\left(1-d \eta^{\beta-1}\right) t^{\beta-2}}{\nabla} \int_{0}^{\xi} \frac{(\xi-s)^{\beta-1}}{\Gamma(\beta)} q(s) \Psi(s) d s,
\end{aligned}
$$




$$
\begin{aligned}
& M_{1}=\max \left\{\frac{v_{1}+\mu_{1}}{\Delta}\left\|\Psi_{1}\right\|_{1} C_{1}+\frac{\omega_{1}+\lambda_{1}}{\Delta}\left\|\Phi_{1}\right\|_{1} B_{1}\right. \\
& +b_{1} \frac{\left[\Delta+(1+b)\left(v_{1}+\mu_{1}\right)+a\left(\lambda_{1}+\omega_{1}\right)\right] \mathbf{B}\left(\alpha+l_{1}, k_{1}+1\right)}{\Gamma(\alpha) \Delta}, \\
& \frac{v_{1} \frac{\Gamma(\alpha)}{\Gamma(\alpha-m)}+\mu_{1} \frac{\Gamma(\alpha-1)}{\Gamma(\alpha-m-1)}}{\Delta}\left\|\Psi_{1}\right\|_{1} C_{1}+\frac{\omega_{1} \frac{\Gamma(\alpha-1)}{\Gamma(\alpha-m-1)}+\lambda_{1} \frac{\Gamma(\alpha)}{\Gamma(\alpha-m)}}{\Delta}\left\|\Phi_{1}\right\|_{1} B_{1} \\
& +a_{1} \frac{\left[v_{1} \frac{\Gamma(\alpha)}{\Gamma(\alpha-m)}+\mu_{1} \frac{\Gamma(\alpha-1)}{|\Gamma(\alpha-m-1)|}+\left(b v_{1} \frac{\Gamma(\alpha)}{\Gamma(\alpha-m)}+b \mu_{1} \frac{\Gamma(\alpha-1)}{|\Gamma(\alpha-m-1)|}\right) \eta^{\alpha+k_{1}+l_{1}}\right] \mathbf{B}\left(\alpha-m+l_{1}, k_{1}+1\right)}{\Gamma(\alpha) \Delta} \\
& \left.+b_{1} \frac{\left(a \lambda_{1} \frac{\Gamma(\alpha)}{\Gamma(\alpha-m)}+a \omega_{1} \frac{\Gamma(\alpha-1)}{|\Gamma(\alpha-m-1)|}\right) \xi^{\alpha+k_{1}+l_{1}} \mathbf{B}\left(\alpha-m+l_{1}, k_{1}+1\right)}{\Gamma(\alpha) \Delta}+\frac{b_{1} \mathbf{B}\left(\alpha-m+l_{1}, k_{1}+1\right)}{\Gamma(\alpha-m)}\right\} \\
& N_{1}=\max \left\{\frac{v_{1}+\mu_{1}}{\Delta}\left\|\Psi_{1}\right\|_{1} D_{1}+\frac{\omega_{1}+\lambda_{1}}{\Delta}\left\|\Phi_{1}\right\|_{1} A_{1}\right. \\
& +a_{1} \frac{\left[\Delta+(1+b)\left(v_{1}+\mu_{1}\right)+a\left(\lambda_{1}+\omega_{1}\right)\right] \mathbf{B}\left(\alpha+l_{1}, k_{1}+1\right)}{\Gamma(\alpha) \Delta}, \\
& \frac{v_{1} \frac{\Gamma(\alpha)}{\Gamma(\alpha-m)}+\mu_{1} \frac{\Gamma(\alpha-1)}{|\Gamma(\alpha-m-1)|}}{\Delta}\left\|\Psi_{1}\right\|_{1} D_{1}+\frac{\omega_{1} \frac{\Gamma(\alpha-1)}{|\Gamma(\alpha-m-1)|}+\lambda_{1} \frac{\Gamma(\alpha)}{\Gamma(\alpha-m)}}{\Delta}\left\|\Phi_{1}\right\|_{1} A_{1} \\
& +b_{1} \frac{\left[v_{1} \frac{\Gamma(\alpha)}{\Gamma(\alpha-m)}+\mu_{1} \frac{\Gamma(\alpha-1)}{\Gamma(\alpha-m-1) \mid}+\left(b v_{1} \frac{\Gamma(\alpha)}{\Gamma(\alpha-m)}+b \mu_{1} \frac{\Gamma(\alpha-1)}{|\Gamma(\alpha-m-1)|}\right) \eta^{\alpha+k_{1}+l_{1}}\right] \mathbf{B}\left(\alpha-m+l_{1}, k_{1}+1\right)}{\Gamma(\alpha) \Delta} \\
& \left.+a_{1} \frac{\left(a \lambda_{1} \frac{\Gamma(\alpha)}{\Gamma(\alpha-m)}+a \omega_{1} \frac{\Gamma(\alpha-1)}{\Gamma(\alpha-m-1) \mid}\right) \xi^{\alpha+k_{1}+l_{1}} \mathbf{B}\left(\alpha-m+l_{1}, k_{1}+1\right)}{\Gamma(\alpha) \Delta}+\frac{a_{1} \mathbf{B}\left(\alpha-m+l_{1}, k_{1}+1\right)}{\Gamma(\alpha-m)}\right\}, \\
& M_{2}=\max \left\{\frac{v_{2}+\mu_{2}}{\nabla}\left\|\Psi_{2}\right\|_{1} C_{2}+\frac{\omega_{2}+\lambda_{2}}{\nabla}\left\|\Phi_{2}\right\|_{1} B_{2}\right. \\
& +b_{2} \frac{\left[\nabla+(1+d)\left(v_{2}+\mu_{2}\right)+c\left(\lambda_{2}+\omega_{2}\right)\right] \mathbf{B}\left(\beta+l_{2}, k_{2}+1\right)}{\Gamma(\beta) \nabla}, \\
& \frac{v_{2} \frac{\Gamma(\beta)}{\Gamma(\beta-n)}+\mu_{2} \frac{\Gamma(\beta-1)}{\Gamma(\beta-n-1) \mid}}{\nabla}\left\|\Psi_{2}\right\|_{1} C_{2}+\frac{\omega_{2} \frac{\Gamma(\beta-1)}{|\Gamma(\beta-m-1)|}+\lambda_{2} \frac{\Gamma(\beta)}{\Gamma(\beta-n)}}{\nabla}\left\|\Phi_{2}\right\|_{1} B_{2} \\
& +a_{2} \frac{\left[v_{2} \frac{\Gamma(\beta)}{\Gamma(\beta-n)}+\mu_{2} \frac{\Gamma(\beta-1)}{|\Gamma(\beta-n-1)|}+\left(d v_{2} \frac{\Gamma(\beta)}{\Gamma(\beta-n)}+d \mu_{2} \frac{\Gamma(\beta-1)}{|\Gamma(\beta-n-1)|}\right) \eta^{\beta+k_{2}+l_{2}}\right] \mathbf{B}\left(\beta-n+l_{2}, k_{2}+1\right)}{\Gamma(\beta) \nabla} \\
& \left.+b_{2} \frac{\left(c \lambda_{2} \frac{\Gamma(\beta)}{\Gamma(\beta-n)}+c \omega_{2} \frac{\Gamma(\beta-1)}{\Gamma(\beta-n-1) \mid}\right) \xi^{\beta+k_{2}+l_{2}} \mathbf{B}\left(\beta-n+l_{2}, k_{2}+1\right)}{\Gamma(\beta) \nabla}+\frac{b_{2} \mathbf{B}\left(\beta-n+l_{2}, k_{2}+1\right)}{\Gamma(\beta-n)}\right\}
\end{aligned}
$$

and

$$
\begin{aligned}
& N_{2}=\max \left\{\frac{v_{2}+\mu_{2}}{\nabla}\left\|\Psi_{2}\right\|_{1} D_{2}+\frac{\omega_{2}+\lambda_{2}}{\nabla}\left\|\Phi_{2}\right\|_{1} A_{2}\right. \\
& +a_{2} \frac{\left[\nabla+(1+d)\left(v_{2}+\mu_{2}\right)+c\left(\lambda_{2}+\omega_{2}\right)\right] \mathbf{B}\left(\beta+l_{2}, k_{2}+1\right)}{\Gamma(\beta) \nabla}, \\
& \frac{v_{2} \frac{\Gamma(\beta)}{\Gamma(\beta-n)}+\mu_{2} \frac{\Gamma(\beta-1)}{|\Gamma(\beta-n-1)|}}{\nabla}\left\|\Psi_{2}\right\|_{1} D_{2}+\frac{\omega_{2} \frac{\Gamma(\beta-1)}{\Gamma(\beta-n-1) \mid}+\lambda_{2} \frac{\Gamma(\beta)}{\Gamma(\beta-n)}}{\nabla}\left\|\Phi_{2}\right\|_{1} A_{2} \\
& +b_{2} \frac{\left[v_{2} \frac{\Gamma(\beta)}{\Gamma(\beta-n)}+\mu_{2} \frac{\Gamma(\beta-1)}{\Gamma(\beta-n-1) \mid}+\left(d v_{2} \frac{\Gamma(\beta)}{\Gamma(\beta-n)}+d \mu_{2} \frac{\Gamma(\beta-1)}{\Gamma(\beta-n-1) \mid}\right) \eta^{\left.\beta+k_{2}+l_{2}\right] \mathbf{B}\left(\beta-n+l_{2}, k_{2}+1\right)}\right.}{\Gamma(\beta) \nabla} \\
& \left.+a_{2} \frac{\left(c \lambda_{2} \frac{\Gamma(\beta)}{\Gamma(\beta-n)}+c \omega_{2} \frac{\Gamma(\beta-1)}{|\Gamma(\beta-n-1)|}\right) \xi^{\beta+k_{2}+l_{2} \mathbf{B}\left(\beta-n+l_{2}, k_{2}+1\right)}}{\Gamma(\beta) \nabla}+\frac{a_{2} \mathbf{B}\left(\beta-n+l_{2}, k_{2}+1\right)}{\Gamma(\beta-n)}\right\} .
\end{aligned}
$$


Denote

$$
\begin{aligned}
& M=M_{1}+N_{1}, N=M_{2}+N_{2}, \\
& \Phi_{0}=\max \left\{\|\bar{\Phi}\|_{1}, 1\right\}, \quad \Psi_{0}=\max \left\{\|\bar{\Psi}\|_{1}, 1\right\}, \\
& \tau=\max \left\{\epsilon_{1}, \delta_{1}\right\}, \quad \sigma=\max \left\{\sigma_{1}, \gamma_{1}\right\} .
\end{aligned}
$$

3.1. Theorem. Suppose that $\Delta>0, \nabla>0, b \eta^{\alpha-1} \leq 1, d \xi^{\alpha-1} \leq 1$, (i)-(iv) defined in Section 1 and (B1) hold. Then BVP(1.8) has at least one positive solution if one of the followings is satisfied:

(I) $\tau \sigma<1$

(II) $\tau \sigma=1$ with $N M^{1 / \sigma}<1$ or $M N^{1 / \tau}<1$

(III) $\tau \sigma>1$ with

$$
\frac{M(\tau \sigma-1) \tau \sigma\left[M \Psi_{0}+\Phi_{0}\right]^{\tau \sigma-1}}{(\tau \sigma-1)^{\tau \sigma}} \leq \frac{1}{N^{\sigma}} \text { or } \frac{N(\tau \sigma-1) \tau \sigma\left[N \Phi_{0}+\Psi_{0}\right]^{\tau \sigma-1}}{(\tau \sigma-1)^{\tau \sigma}} \leq \frac{1}{M^{\tau}}
$$

Proof. From Lemmas 2.6 and 2.7, we know that $(x, y)$ is a solution of $\operatorname{BVP}(1.8)$ if and only if $(x, y)$ is a fixed point of $T$. From Lemma 2.8, $T: X \times Y \rightarrow X \times Y$ is completely continuous. By Lemma 2.9 and (i)-(iv), $(x, y)$ is a positive solution of $\operatorname{BVP}(1.8)$ if and only if $(x, y)$ is a fixed point of $T$.

To get a fixed point of $T$, we apply the Schauder's fixed point theorem. We should define an closed convex bounded subset $\Omega$ of $E$ such that $T(\Omega) \subseteq \Omega$.

It is easy to see that $\bar{\Phi} \in X, \bar{\Psi} \in Y$. For $r_{1}>0, r_{2}>0$, denote $\Omega=\{(x, y) \in E$ : $\left.\|x-\bar{\Phi}\| \leq r_{1},\|y-\bar{\Psi}\| \leq r_{2}\right\}$. For $(x, y) \in \Omega$, we get

$$
\|x\| \leq\|x-\bar{\Phi}\|+\|\bar{\Phi}\| \leq r_{1}+\|\bar{\Phi}\|, \quad\|y\| \leq\|y-\bar{\Psi}\|+\|\bar{\Psi}\| \leq r_{2}+\|\bar{\Psi}\| .
$$

Furthermore, we have

$$
\begin{aligned}
& \left|f\left(t, y(t), D_{0^{+}}^{n} y(t)\right)-\Phi(t)\right| \leq b_{1}\left|t^{2-\beta} y(t)\right|^{\epsilon_{1}}+a_{1}\left|t^{2+n-\beta} D_{0^{+}}^{n} y(t)\right|^{\delta_{1}}, \\
& \left|g\left(t, x(t), D_{0^{+}}^{m} x(t)\right)-\Psi(t)\right| \leq b_{2}\left|t^{2-\alpha} x(t)\right|^{\sigma_{1}}+a_{2}\left|t^{2+m-\alpha} D_{0^{+}}^{m} x(t)\right|^{\gamma_{1}}, \\
& \left|\phi_{1}\left(t, y(t), D_{0^{+}}^{n} y(t)\right)-\Phi_{10}(t)\right| \leq \Phi_{1}(t)\left[B_{1}\left|t^{2-\beta} y(t)\right|^{\epsilon_{1}}+A_{1}\left|t^{2+n-\beta} D_{0^{+}}^{n} y(t)\right|^{\delta_{1}},\right. \\
& \left|\psi_{1}\left(t, y(t), D_{0^{+}}^{n} y(t)\right)-\Psi_{10}(t)\right| \leq \Psi_{1}(t)\left[C_{1}\left|t^{2-\beta} y(t)\right|^{\epsilon_{1}}+D_{1}\left|t^{2+n-\beta} D_{0^{+}}^{n} y(t)\right|^{\delta_{1}}\right] \\
& \left|\phi_{2}\left(t, x(t), D_{0^{+}}^{m} x(t)\right)-\Phi_{20}(t)\right| \leq \Phi_{2}(t)\left[B_{2}\left|t^{2-\alpha} x(t)\right|^{\sigma_{1}}+A_{2}\left|t^{2+m-\alpha} D_{0^{+}}^{m} x(t)\right|^{\gamma_{1}}\right], \\
& \left|\psi_{2}\left(t, x(t), D_{0^{+}}^{m} x(t)\right)-\Psi_{20}(t)\right| \leq \Psi_{2}(t)\left[C_{2}\left|t^{2-\alpha} x(t)\right|^{\sigma_{1}}+D_{2}\left|t^{2+m-\alpha} D_{0^{+}}^{m} x(t)\right|^{\gamma_{1}}\right]
\end{aligned}
$$

hold for all $t \in(0,1)$. It follows that

$$
\begin{aligned}
& \left|f\left(t, y(t), D_{0^{+}}^{n} y(t)\right)-\Phi(t)\right| \leq b_{1}\|y\|^{\epsilon_{1}}+a_{1}\|y\|^{\delta_{1}}, t \in(0,1), \\
& \left|g\left(t, x(t), D_{0^{+}}^{m} x(t)\right)-\Psi(t)\right| \leq b_{2}\|x\|^{\sigma_{1}}+a_{2}\|x\|^{\gamma_{1}}, t \in(0,1), \\
& \left|\phi_{1}\left(t, y(t), D_{0^{+}}^{n} y(t)\right)-\Phi_{10}(t)\right| \leq \Phi_{1}(t)\left[B_{1}\|y\|^{\epsilon_{1}}+A_{1}\|y\|^{\delta_{1}}\right], t \in(0,1), \\
& \left|\psi_{1}\left(t, y(t), D_{0^{+}}^{n} y(t)\right)-\Psi_{10}(t)\right| \leq \Psi_{1}(t)\left[C_{1}\|y\|^{\epsilon_{1}}+D_{1}\|y\|^{\delta_{1}}\right], t \in(0,1), \\
& \left|\phi_{2}\left(t, x(t), D_{0^{+}}^{m} x(t)\right)-\Phi_{20}(t)\right| \leq \Phi_{2}(t)\left[B_{2}\|x\|^{\sigma_{1}}+A_{2}\|x\|^{\gamma_{1}}\right], t \in(0,1), \\
& \left|\psi_{2}\left(t, x(t), D_{0^{+}}^{m} x(t)\right)-\Psi_{20}(t)\right| \leq \Psi_{2}(t)\left[C_{2}\|x\|^{\sigma_{1}}+D_{2}\|x\|^{\gamma_{1}}\right], t \in(0,1) .
\end{aligned}
$$


By the definition of $T$, we have

$$
\begin{aligned}
& t^{2-\alpha}\left|\left(T_{1} y\right)(t)-\bar{\Phi}(t)\right| \\
& \leq \frac{v_{1}+\mu_{1}}{\Delta}\left\|\Psi_{1}\right\|_{1}\left[C_{1}\|y\|^{\epsilon_{1}}+D_{1}\|y\|^{\delta_{1}}\right]+\frac{\omega_{1}+\lambda_{1}}{\Delta}\left\|\Phi_{1}\right\|_{1}\left[\left.B_{1}\|y\|\right|^{\epsilon_{1}}+A_{1}\|y\|^{\delta_{1}}\right] \\
& +t^{2-\alpha} \int_{0}^{t} \frac{(t-s)^{\alpha-1}}{\Gamma(\alpha)} s^{k_{1}}(1-s)^{l_{1}} d s\left[b_{1}\|y\|^{\epsilon_{1}}+a_{1}\|y\|^{\delta_{1}}\right] \\
& +\frac{v_{1}+\mu_{1}}{\Delta} \int_{0}^{1} \frac{(1-s)^{\alpha-1}}{\Gamma(\alpha)} s^{k_{1}}(1-s)^{l_{1}} d s\left[b_{1}\|y\|^{\epsilon_{1}}+a_{1}\|y\|^{\delta_{1}}\right] \\
& +\frac{b v_{1}+b \mu_{1}}{\Delta} \int_{0}^{\eta} \frac{(\eta-s)^{\alpha-1}}{\Gamma(\alpha)} s^{k_{1}}(1-s)^{l_{1}} d s\left[b_{1}\|y\|^{\epsilon_{1}}+a_{1}\|y\|^{\delta_{1}}\right] \\
& +\frac{a \lambda_{1}+a \omega_{1}}{\Delta} \int_{0}^{\xi} \frac{(\xi-s)^{\alpha-1}}{\Gamma(\alpha)} s^{k_{1}}(1-s)^{l_{1}} d s\left[b_{1}\|y\|^{\epsilon_{1}}+a_{1}\|y\|^{\delta_{1}}\right]
\end{aligned}
$$

$$
\begin{aligned}
& \leq\left(\frac{v_{1}+\mu_{1}}{\Delta}\left\|\Psi_{1}\right\|_{1} C_{1}+\frac{\omega_{1}+\lambda_{1}}{\Delta}\left\|\Phi_{1}\right\|_{1} B_{1}\right. \\
& \left.+b_{1} \frac{\left[\Delta+(1+b)\left(v_{1}+\mu_{1}\right)+a\left(\lambda_{1}+\omega_{1}\right)\right] \mathbf{B}\left(\alpha+l_{1}, k_{1}+1\right)}{\Gamma(\alpha) \Delta}\right)\|y\|^{\epsilon_{1}} \\
& +\left(\frac{v_{1}+\mu_{1}}{\Delta}\left\|\Psi_{1}\right\|_{1} D_{1}+\frac{\omega_{1}+\lambda_{1}}{\Delta}\left\|\Phi_{1}\right\|_{1} A_{1}\right. \\
& \left.+a_{1} \frac{\left[\Delta+(1+b)\left(v_{1}+\mu_{1}\right)+a\left(\lambda_{1}+\omega_{1}\right)\right] \mathbf{B}\left(\alpha+l_{1}, k_{1}+1\right)}{\Gamma(\alpha) \Delta}\right)\|y\|^{\delta_{1}}
\end{aligned}
$$


and similarly we get

$$
\begin{aligned}
& t^{2+m-\alpha}\left|D_{0^{+}}^{m}\left(T_{1} y\right)(t)-D_{0^{+}}^{m} \bar{\Phi}(t)\right| \\
& \leq \frac{v_{1} \frac{\Gamma(\alpha)}{\Gamma(\alpha-m)}+\mu_{1} \frac{\Gamma(\alpha-1)}{\Gamma(\alpha-m-1)}}{\Delta}\left\|\Psi_{1}\right\|_{1}\left[C_{1}\|y\|^{\epsilon_{1}}+D_{1}\|y\|^{\delta_{1}}\right] \\
& +\frac{\omega_{1} \frac{\Gamma(\alpha-1)}{\Gamma(\alpha-m-1)}+\lambda_{1} \frac{\Gamma(\alpha)}{\Gamma(\alpha-m)}}{\Delta}\left\|\Phi_{1}\right\|_{1}\left[B_{1}\|y\|^{\epsilon_{1}}+A_{1}\|y\|^{\delta_{1}}\right] \\
& +t^{2+m-\alpha} \int_{0}^{t} \frac{(t-s)^{\alpha-m-1}}{\Gamma(\alpha-m)} s^{k_{1}}(1-s)^{l_{1}} d s\left[b_{1}\|y\|^{\epsilon_{1}}+a_{1}\|y\|^{\delta_{1}}\right.
\end{aligned}
$$

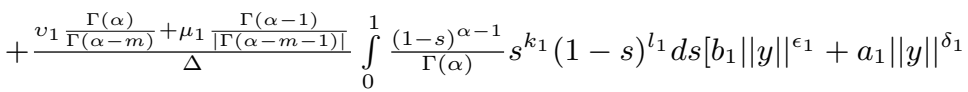

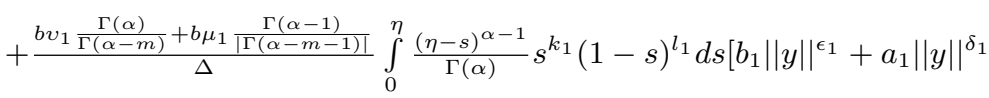

$$
\begin{aligned}
& +\frac{a \lambda_{1} \frac{\Gamma(\alpha)}{\Gamma(\alpha-m)}+a \omega_{1} \frac{\Gamma(\alpha-1)}{|\Gamma(\alpha-m-1)|}}{\Delta} \int_{0}^{\xi} \frac{(\xi-s)^{\alpha-1}}{\Gamma(\alpha)} s^{k_{1}}(1-s)^{l_{1}} d s\left[b_{1}\|y\|^{\epsilon_{1}}+a_{1}\|y\|^{\delta_{1}}\right. \\
& \leq\left(\frac{v_{1} \frac{\Gamma(\alpha)}{\Gamma(\alpha-m)}+\mu_{1} \frac{\Gamma(\alpha-1)}{\Gamma(\alpha-m-1)}}{\Delta}\left\|\Psi_{1}\right\|_{1} C_{1}+\frac{\omega_{1} \frac{\Gamma(\alpha-1)}{\Gamma(\alpha-m-1)}+\lambda_{1} \frac{\Gamma(\alpha)}{\Gamma(\alpha-m)}}{\Delta}\left\|\Phi_{1}\right\|_{1} B_{1}\right. \\
& +\frac{b_{1} \mathbf{B}\left(\alpha-m+l_{1}, k_{1}+1\right)}{\Gamma(\alpha-m)} \\
& +a_{1} \frac{\left[v_{1} \frac{\Gamma(\alpha)}{\Gamma(\alpha-m)}+\mu_{1} \frac{\Gamma(\alpha-1)}{\Gamma(\alpha-m-1) \mid}+\left(b v_{1} \frac{\Gamma(\alpha)}{\Gamma(\alpha-m)}+b \mu_{1} \frac{\Gamma(\alpha-1)}{\Gamma(\alpha-m-1)}\right) \eta^{\left.\alpha+k_{1}+l_{1}\right] \mathbf{B}\left(\alpha-m+l_{1}, k_{1}+1\right)}\right.}{\Gamma(\alpha) \Delta} \\
& \left.+b_{1} \frac{\left(a \lambda_{1} \frac{\Gamma(\alpha)}{\Gamma(\alpha-m)}+a \omega_{1} \frac{\Gamma(\alpha-1)}{\Gamma(\alpha-m-1)}\right) \xi^{\alpha+k_{1}+l_{1}} \mathbf{B}\left(\alpha-m+l_{1}, k_{1}+1\right)}{\Gamma(\alpha) \Delta}\right)\|y\|^{\epsilon_{1}} \\
& +\left(\frac{v_{1} \frac{\Gamma(\alpha)}{\Gamma(\alpha-m)}+\mu_{1} \frac{\Gamma(\alpha-1)}{\Gamma(\alpha-m-1)}}{\Delta}\left\|\Psi_{1}\right\|_{1} D_{1}+\frac{\omega_{1} \frac{\Gamma(\alpha-1)}{\Gamma(\alpha-m-1)}+\lambda_{1} \frac{\Gamma(\alpha)}{\Gamma(\alpha-m)}}{\Delta}\left\|\Phi_{1}\right\|_{1} A_{1}\right. \\
& +\frac{a_{1} \mathbf{B}\left(\alpha-m+l_{1}, k_{1}+1\right)}{\Gamma(\alpha-m)} \\
& +b_{1} \frac{\left[v_{1} \frac{\Gamma(\alpha)}{\Gamma(\alpha-m)}+\mu_{1} \frac{\Gamma(\alpha-1)}{|\Gamma(\alpha-m-1)|}+\left(b v_{1} \frac{\Gamma(\alpha)}{\Gamma(\alpha-m)}+b \mu_{1} \frac{\Gamma(\alpha-1)}{|\Gamma(\alpha-m-1)|}\right) \eta^{\alpha+k_{1}+l_{1}}\right] \mathbf{B}\left(\alpha-m+l_{1}, k_{1}+1\right)}{\Gamma(\alpha) \Delta}
\end{aligned}
$$

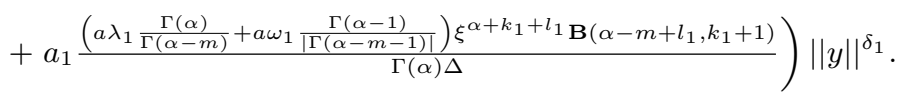

We get

$$
\left\|T_{1} y-\bar{\Phi}\right\| \leq M_{1}\left(r_{2}+\|\bar{\Psi}\|_{1}\right)^{\epsilon_{1}}+N_{1}\left[r_{2}+\|\bar{\Psi}\|_{1}\right]^{\delta_{1}} \leq M\left[r_{2}+\Psi_{0}\right]^{\tau} .
$$

Similarly we get

$$
\left\|T_{2} x-\bar{\Psi}\right\| \leq M_{2}\left[r_{1}+\|\bar{\Phi}\|\right]^{\sigma_{1}}+N_{2}\left[+\left[r_{1}+\|\bar{\Phi}\|\right]^{\gamma_{1}} \leq N\left[r_{1}+\Phi_{0}\right]^{\sigma} .\right.
$$

If there exists $r_{1}, r_{2}>0$ such that

$$
M\left[r_{2}+\Psi_{0}\right]^{\tau} \leq r_{1}, \quad N\left[r_{1}+\Phi_{0}\right]^{\sigma} \leq r_{2},
$$


we let $\Omega=\left\{(x, y) \in E:\left\|x-\Phi_{1}\right\| \leq r_{1},\left\|y-\Phi_{2}\right\| \leq r_{2}\right\}$, then we get $T(\Omega) \subset \Omega$. Hence the Schauder's fixed point theorem implies that $T$ has a fixed point $(x, y) \in \Omega$. So $(x, y)$ is a solution of $\mathrm{BVP}(1.8)$.

Now we will prove that (3.2) has positive solution $r_{1}, r_{2}>0$. We transform (3.2) to the following inequalities:

$$
r_{2} \leq\left(\frac{r_{1}}{M}-\Psi_{0}\right)^{1 / \sigma}, r_{1} \leq\left(\frac{r_{2}}{N}-\Phi_{0}\right)^{1 / \tau}
$$

Hence we get

$$
N\left(r_{1}+\Phi_{0}\right)^{\tau} \leq r_{2} \leq\left(\frac{r_{1}}{M}-\Psi_{0}\right)^{1 / \sigma}
$$

or

$$
M\left(r_{2}+\Psi_{0}\right)^{\sigma} \leq r_{1} \leq\left(\frac{r_{2}}{N}-\Phi_{0}\right)^{1 / \tau}
$$

Case (i) $\sigma \tau<1$.

It is easy to see that there exists $r_{1}>0$ sufficiently large such that $N\left(r_{1}+\Phi_{0}\right)^{\tau} \leq$ $\left(\frac{r_{1}}{M}-\Psi_{0}\right)^{1 / \sigma}$. Then we can choose $r_{2}$ satisfying $N\left(r_{1}+\Phi_{0}\right)^{\tau} \leq r_{2} \leq\left(\frac{r_{1}}{M}-\Psi_{0}\right)^{1 / \sigma}$.

Hence (3.2) has positive solution $r_{1}>0, r_{2}>0$. We choose $\Omega=\{(x, y) \in E$ : $\left.\|x-\bar{\Phi}\| \leq r_{1},\|y-\bar{\Psi}\| \leq r_{2}\right\}$. Then we get $T(\Omega) \subset \Omega$. Hence the Schauder's fixed point theorem implies that $T$ has a fixed point $(x, y) \in \Omega$. So $(x, y)$ is a positive solution of $\operatorname{BVP}(1.8)$.

Case (ii) $\sigma \tau=1$.

If $N M^{1 / \sigma}<1$, then

$$
\lim _{r \rightarrow+\infty} \frac{N\left(r_{1}+\Phi_{0}\right)^{\tau}}{\left(\frac{r_{1}}{M}-\Psi_{0}\right)^{1 / \sigma}}=N M^{1 / \sigma}<1
$$

So there exists $r_{1}>0$ sufficiently large such that $N\left(r_{1}+\Phi_{0}\right)^{\tau} \leq\left(\frac{r_{1}}{M}-\Psi_{0}\right)^{1 / \sigma}$. Then we can choose $r_{2}$ satisfying $N\left(r_{1}+\Phi_{0}\right)^{\tau} \leq r_{2} \leq\left(\frac{r_{1}}{M}-\Psi_{0}\right)^{1 / \sigma}$.

If $M N^{1 / \tau}<1$, then there exists $r_{2}>0$ sufficiently large such that $M\left(r_{2}+\Psi_{0}\right)^{\sigma} \leq$ $\left(\frac{r_{2}}{N}-\Phi_{0}\right)^{1 / \tau}$. Then we can choose $r_{1}$ satisfying $M\left(r_{2}+\Psi_{0}\right)^{\sigma} \leq r_{1} \leq\left(\frac{r_{2}}{N}-\Phi_{0}\right)^{1 / \tau}$.

Hence (3.2) has positive solution $r_{1}>0, r_{2}>0$. We choose $\Omega=\{(x, y) \in E$ : $\left.\|x-\bar{\Phi}\| \leq r_{1},\|y-\bar{\Psi}\| \leq r_{2}\right\}$. Then we get $T(\Omega) \subset \Omega$. Hence the Schauder's fixed point theorem implies that $T$ has a fixed point $(x, y) \in \Omega$. So $(x, y)$ is a positive solution of $\operatorname{BVP}(1.8)$.

Case (iii) $\sigma \tau>1$.

If

$$
\frac{M(\tau \sigma-1) \tau \sigma\left[M \Psi_{0}+\Phi_{0}\right]^{\tau \sigma-1}}{(\tau \sigma-1)^{\tau \sigma}} \leq \frac{1}{N^{\sigma}},
$$

then let $r_{1}=\frac{\tau \sigma M \Psi_{0}+\Phi_{0}}{\tau \sigma-1}$. It is easy to see that $N\left(r_{1}+\Phi_{0}\right)^{\tau} \leq\left(\frac{r_{1}}{M}-\Psi_{0}\right)^{1 / \sigma}$. Then we can choose $r_{2}$ satisfying $N\left(r_{1}+\Phi_{0}\right)^{\tau} \leq r_{2} \leq\left(\frac{r_{1}}{M}-\Psi_{0}\right)^{1 / \sigma}$.

If

$$
\frac{N(\tau \sigma-1) \tau \sigma\left[N \Phi_{0}+\Psi_{0}\right]^{\tau \sigma-1}}{(\tau \sigma-1)^{\tau \sigma}} \leq \frac{1}{M^{\tau}}
$$

then let $r_{2}=\frac{\tau \sigma N \Phi_{0}+\Psi_{0}}{\tau \sigma-1}$. It is easy to see that $M\left(r_{2}+\Psi_{0}\right)^{\sigma} \leq\left(\frac{r_{2}}{N}-\Phi_{0}\right)^{1 / \tau}$. Then we can choose $r_{1}$ satisfying $M\left(r_{2}+\Psi_{0}\right)^{\sigma} \leq r_{1} \leq\left(\frac{r_{2}}{N}-\Phi_{0}\right)^{1 / \tau}$.

Hence (3.2) has positive solution $r_{1}>0, r_{2}>0$. We choose $\Omega=\{(x, y) \in E$ : $\left.\|x-\bar{\Phi}\| \leq r_{1},\|y-\bar{\Psi}\| \leq r_{2}\right\}$. Then we get $T(\Omega) \subset \Omega$. Hence the Schauder's fixed point theorem implies that $T$ has a fixed point $(x, y) \in \Omega$. So $(x, y)$ is a positive solution of $\operatorname{BVP}(1.8)$.

The proof of Theorem 3.1 is complete. 
3.2. Remark. If (B1) holds with $\max \left\{\epsilon_{1}, \delta_{1}\right\} \max \left\{\sigma_{1}, \gamma_{1}\right\} \geq 1$, it is easy to see that all known results in [1, 17] can not be applied to establish existence results for solutions of $\operatorname{BVP}(1.8)$. It is easy to see that $\lim M=\lim N=0$ for sufficiently small $a_{i}, b_{i}, C_{i}, D_{i}, A_{i}, B_{i}(i=1,2)$. Then

$$
\begin{aligned}
& N M^{1 / \sigma}<1, \quad M N^{1 / \tau}<1 \\
& \frac{M(\tau \sigma-1) \tau \sigma\left[M \Psi_{0}+\Phi_{0}\right]^{\tau \sigma-1}}{(\tau \sigma-1)^{\tau \sigma}} \leq \frac{1}{N^{\sigma}} \text { and } \frac{N(\tau \sigma-1) \tau \sigma\left[N \Phi_{0}+\Psi_{0}\right]^{\tau \sigma-1}}{(\tau \sigma-1)^{\tau \sigma}} \leq \frac{1}{M^{\tau}} .
\end{aligned}
$$

hold for sufficiently small $a_{i}, b_{i}, C_{i}, D_{i}, A_{i}, B_{i}(i=1,2)$. From Theorem $3.1, \operatorname{BVP}(1.8)$ has at least one solution for $\sigma \tau<1$, and for sufficiently small $a_{i}, b_{i}, C_{i}, D_{i}, A_{i}, B_{i}(i=1,2)$ when $\sigma \tau \geq 1$.

\section{Numerical examples}

In this section, we present two examples for the illustration of our main result (Theorem 3.1).

4.1. Example. We consider the following boundary value problem

$$
\left\{\begin{array}{l}
D_{0^{+}}^{\frac{19}{10}} u(t)+t^{-\frac{1}{10}}(1-t)^{-\frac{21}{20}} f\left(t, v(t), D_{0^{+}}^{\frac{13}{20}} v(t)\right)=0, \quad t \in(0,1), \\
D_{0^{+}}^{\frac{39}{20}} v(t)+t^{-\frac{1}{10}}(1-t)^{-\frac{23}{20}} g\left(t, u(t), D_{0^{+}}^{\frac{4}{5}} u(t)\right)=0, \quad t \in(0,1) \\
\lim _{t \rightarrow 0} t^{\frac{1}{5}} u(t)-\frac{1}{2} u(1 / 2)=0 \\
u(1)-\frac{1}{2} u(3 / 4)=0 \\
\lim _{t \rightarrow 0} t^{\frac{1}{9}} v(t)-\frac{1}{2} v(1 / 2)=0 \\
v(1)-\frac{1}{2} v(3 / 4)=0
\end{array}\right.
$$

Then

(i) $\operatorname{BVP}(4.1)$ has at least one positive solution if there exists a constant $H>0$ such that

$$
\begin{aligned}
& \left|f(t, u, v)-t^{2}\right| \leq H, \quad t \in(0,1), u, v \in \mathbb{R} \\
& \left|g(t, u, v)-t^{5}\right| \leq H, \quad t \in(0,1), u, v \in \mathbb{R}
\end{aligned}
$$

(ii) $\operatorname{BVP}(4.1)$ has at least one positive solution if

$$
\begin{aligned}
& \left|f(t, u, v)-t^{2}\right| \leq b_{1} t^{\frac{\epsilon_{1}}{20}} u^{\epsilon_{1}}, b_{1} \geq 0, \epsilon_{1}>0, \\
& \left|g(t, u, v)-t^{5}\right| \leq b_{2} t^{\frac{\sigma_{1}}{10}} u^{\sigma_{1}}, b_{2} \geq 0, \sigma_{1}>0
\end{aligned}
$$

and one of the followings holds:

(a) $\epsilon_{1} \sigma_{1}<1$;

(b) $\epsilon_{1} \sigma_{1}=1$ with $\left(38.1089 b_{1}\right)^{1 / \sigma_{1}} 34.0678 b_{2}<1$ or $38.1089 b_{1}\left(34.0678 b_{2}\right)^{1 / \tau_{1}}<1$

(c) $\epsilon_{1} \sigma_{1}>1$ with

$$
\frac{38.1089 b_{1}\left(\epsilon_{1} \sigma_{1}-1\right) \epsilon_{1} \sigma_{1}\left[38.1089 b_{1} \Psi_{0}+\Phi_{0}\right]^{\epsilon_{1} \sigma_{1}-1}}{\left(\epsilon_{1} \sigma_{1}-1\right)^{\epsilon_{1} \sigma_{1}}}\left(34.0678 b_{2}\right)^{\sigma_{1}} \leq 1
$$

or

$$
\frac{34.0678 b_{2}\left(\epsilon_{1} \sigma_{1}-1\right) \epsilon_{1} \sigma_{1}\left[34.0678 b_{2} \Phi_{0}+\Psi_{0}\right]^{\epsilon_{1} \sigma_{1}-1}}{\left(\epsilon_{1} \sigma_{1}-1\right)^{\epsilon_{1} \sigma_{1}}}\left(38.1089 b_{1}\right)^{\epsilon_{1}} \leq 1 .
$$

(iii) $\operatorname{BVP}(4.1)$ has at least one positive solution if

$$
\begin{aligned}
& f(t, u, v)=t^{2}+b_{1} t^{\frac{\epsilon_{1}}{20}} u^{\epsilon_{1}}+a_{1} t^{\frac{7 \delta_{1}}{10}} v^{\delta_{1}}, a_{1}, b_{1} \geq 0, \epsilon_{1}, \delta_{1}>0, \\
& g(t, u, v)=t^{5}+b_{2} t^{\frac{\sigma_{1}}{10}} u^{\sigma_{1}}+a_{2} t^{\frac{9 \gamma_{1}}{10}} v^{\gamma_{1}}, a_{2}, b_{2} \geq 0, \sigma_{1}, \gamma_{1}>0 .
\end{aligned}
$$

with $a_{i}, b_{i}(i=1,2)$ sufficiently small. 
Proof. Corresponding to $\mathrm{BVP}(1.8)$, we have $\alpha=\frac{19}{10}, \beta=\frac{39}{20}, m=\frac{4}{5}$ and $n=\frac{13}{20}$, $\xi=\frac{1}{2}, \eta=\frac{3}{4}, a=b=c=d=\frac{1}{2}$ and $\phi_{i}(t, u, v)=\psi_{i}(t, u, v) \equiv 0(i=1,2)$ and $p(t)=t^{-\frac{1}{10}}(1-t)^{-\frac{21}{20}}, q(t)=t^{-\frac{1}{10}}(1-t)^{-\frac{23}{20}}$.

It is easy to see that (i)-(iv) hold with $k_{1}=-\frac{1}{10}=k_{2}$, and $l_{1}=-\frac{21}{20}, l_{2}=-\frac{23}{20}$. One sees that $k_{1}>-1, \alpha-m+l_{1}>0,2+k_{1}+l_{1}>0, k_{2}>-1, \beta-n+l_{2}>0,2+k_{2}+l_{2}>0$. One sees that both $p$ and $q$ are not integrable on $(0,1)$. Hence (i)-(iv) defined in Section 1 hold.

By direct calculation using Matlab7, we find that

$$
\begin{aligned}
& \mu_{1}=\frac{1}{2}\left(\frac{1}{2}\right)^{\frac{9}{10}} \approx 0.2679, \quad v_{1}=1-\frac{1}{2} \sqrt[10]{2} \approx 0.0670 \\
& \omega_{1}=1-\frac{1}{2}\left(\frac{3}{4}\right)^{\frac{9}{10}} \approx 0.6141, \quad \lambda_{1}=1-\frac{1}{2} \sqrt[10]{2} \approx 0.4641 \\
& \mu_{2}=\frac{1}{2}\left(\frac{1}{2}\right)^{\frac{19}{20}} \approx 0.2588, \quad v_{2}=1-\frac{1}{2} \sqrt[20]{2} \approx 0.4828 \\
& \omega_{2}=1-\frac{1}{2}\left(\frac{3}{4}\right)^{\frac{19}{20}} \approx 0.6196, \quad \lambda_{2}=1-\frac{1}{2} \sqrt[20]{2} \approx 0.4824 \\
& \Delta=\frac{1}{2}\left(\frac{1}{2}\right)^{\frac{9}{10}}\left(1-\frac{1}{2} \sqrt[10]{2}\right)+\left(1-\frac{1}{2} \sqrt[10]{2}\right)\left(1-\frac{1}{2}\left(\frac{3}{4}\right)^{\frac{9}{10}}\right) \approx 0.4093 \\
& \nabla=\frac{1}{2}\left(\frac{1}{2}\right)^{\frac{19}{20}}\left(1-\frac{1}{2} \sqrt[20]{2}\right)+\left(1-\frac{1}{2} \sqrt[20]{2}\right)\left(1-\frac{1}{2}\left(\frac{3}{4}\right)^{\frac{19}{20}}\right) \approx 0.4237
\end{aligned}
$$

Hence $\Delta>0, \nabla>0, b \eta^{\alpha-1} \leq 1, d \xi^{\alpha-1} \leq 1$.

Choose $\Phi(t)=t^{2}, \Psi(t)=t^{5}$. By direct computation, we find that

$$
\begin{aligned}
& t^{\frac{1}{10}}|\bar{\Phi}(t)|=t \frac{1}{10} \mid-\int_{0}^{t} \frac{(t-s)^{\frac{9}{10}}}{\Gamma(19 / 10)} s^{\frac{19}{10}}(1-s)^{-\frac{21}{20}} d s+\frac{\nu_{1} t^{\frac{9}{10}}+\mu_{1} t^{-\frac{1}{10}}}{\Delta} \frac{\mathbf{B}(17 / 20,29 / 10)}{\Gamma(19 / 10)} \\
& -\frac{\nu_{1} t \frac{9}{10}+\mu_{1} t^{-\frac{1}{10}}}{2 \Delta} \int_{0}^{3 / 4} \frac{\left(\frac{3}{4}-s\right)^{\frac{9}{10}}}{\Gamma(19 / 10)} s^{\frac{19}{10}}(1-s)^{-\frac{21}{20}} d s \\
& +\frac{\lambda_{1} t \frac{9}{10}-\omega_{1} t^{-\frac{1}{10}}}{2 \Delta} \int_{0}^{1 / 2} \frac{\left(\frac{1}{2}-s\right)^{\frac{9}{10}}}{\Gamma(19 / 10)} s^{\frac{19}{10}}(1-s)^{-\frac{21}{20}} d s \mid \\
& \leq\left(1+\frac{3}{2} \frac{\nu_{1}+\mu_{1}}{\Delta}+\frac{1}{2} \frac{\lambda_{1}+\omega_{1}}{\Delta}\right) \frac{\mathbf{B}(17 / 20,29 / 10)}{\Gamma(19 / 10)} \approx 2.3895 \\
& t^{\frac{1}{20}}|\bar{\Psi}(t)|=t^{\frac{1}{20}} \mid-\int_{0}^{t} \frac{(t-s)^{\frac{19}{20}}}{\Gamma(39 / 20)} s^{\frac{49}{10}}(1-s)^{-\frac{23}{20}} d s+\frac{\nu_{2} t^{\frac{19}{20}}+\mu_{2} t^{-\frac{1}{20}}}{\nabla} \frac{\mathbf{B}(4 / 5,59 / 10)}{\Gamma(39 / 20)} \\
& -\frac{\nu_{2} t^{\frac{19}{20}}+\mu_{2} t^{-\frac{1}{20}}}{2 \nabla} \int_{0}^{3 / 4} \frac{\left(\frac{3}{4}-s\right)^{\frac{19}{20}}}{\Gamma(39 / 20)} s^{\frac{49}{10}}(1-s)^{-\frac{23}{20}} d s \\
& +\frac{\lambda_{2} t^{\frac{19}{20}}-\omega_{2} t^{-\frac{1}{20}}}{2 \nabla} \int_{0}^{1 / 2} \frac{\left(\frac{1}{2}-s\right)^{\frac{19}{20}}}{\Gamma(39 / 20)} s^{\frac{49}{10}}(1-s)^{-\frac{23}{20}} d s \mid \\
& \leq\left(1+\frac{3}{2} \frac{\nu_{2}+\mu_{2}}{\nabla}+\frac{1}{2} \frac{\left.\lambda_{2}+\omega_{2}\right)}{\nabla} \frac{\mathbf{B}(4 / 5,59 / 10)}{\Gamma(39 / 20)} \approx 1.4335\right.
\end{aligned}
$$


and

$$
\begin{aligned}
& t^{\frac{9}{10}}\left|D_{0^{+}}^{\frac{4}{5}} \bar{\Phi}(t)\right|=t^{\frac{9}{10}} \mid-\int_{0}^{t} \frac{(t-s) \frac{1}{\Gamma(11 / 10)}}{\Gamma \frac{19}{10}}(1-s)^{-\frac{21}{20}} d s \\
& +\frac{\nu_{1} \frac{\Gamma(19 / 10)}{\Gamma(11 / 10)} t^{\frac{1}{10}}+\mu_{1} \frac{\Gamma(9 / 10)}{\Gamma(1 / 10)} t^{-\frac{9}{10}}}{\Delta} \frac{\mathbf{B}(17 / 20,29 / 10)}{\Gamma(19 / 10)} \\
& -\frac{\nu_{1} \frac{\Gamma(19 / 10)}{\Gamma(11 / 10)} t^{\frac{1}{10}}+\mu_{1} \frac{\Gamma(9 / 10)}{\Gamma(1 / 10)} t^{-\frac{9}{10}}}{2 \Delta} \int_{0}^{3 / 4} \frac{\left(\frac{3}{4}-s\right) \frac{9}{10}}{\Gamma(19 / 10)} s^{\frac{19}{10}}(1-s)^{-\frac{21}{20}} d s \\
& +\frac{\lambda_{1} \frac{\Gamma(19 / 10)}{\Gamma(11 / 10)} t^{\frac{1}{10}}-\omega_{1} \frac{\Gamma(9 / 10)}{\Gamma(1 / 10)} t^{-\frac{9}{10}}}{2 \Delta} \int_{0}^{1 / 2} \frac{\left(\frac{1}{2}-s\right)^{\frac{9}{10}}}{\Gamma(19 / 10)} s^{\frac{19}{10}}(1-s)^{-\frac{21}{20}} d s \mid \\
& \leq \frac{\mathbf{B}(1 / 20,29 / 10)}{\Gamma(11 / 10)}+\left(\frac{3}{2} \frac{\nu_{1} \frac{\Gamma(19 / 10)}{\Gamma(11 / 10)}+\mu_{1} \frac{\Gamma(9 / 10)}{\Gamma(1 / 10)}}{\Delta}+\frac{1}{2} \frac{\lambda_{1} \frac{\Gamma(19 / 10)}{\Gamma(11 / 10)}+\omega_{1} \frac{\Gamma(9 / 10)}{\Gamma(1 / 10)}}{\Delta}\right) \frac{\mathbf{B}(17 / 20,29 / 10)}{\Gamma(19 / 10)} \\
& \approx 20.7609,
\end{aligned}
$$

$$
\begin{gathered}
t^{\frac{7}{10}}\left|D_{0^{+}}^{\frac{13}{20}} \bar{\Psi}(t)\right|=t^{\frac{7}{10}} \mid-\int_{0}^{t} \frac{(t-s)^{\frac{3}{10}}}{\Gamma(13 / 10)} s^{\frac{49}{10}}(1-s)^{-\frac{23}{20}} d s \\
+\frac{\nu_{2} \frac{\Gamma(39 / 20)}{\Gamma(13)} t^{\frac{3}{10}}+\mu_{2} \frac{\Gamma(19 / 20)}{\Gamma(3 / 10)} t^{-\frac{7}{10}} \frac{\mathbf{B}(4 / 5,59 / 10)}{\Gamma(39 / 20)}}{\nabla} \\
-\frac{\nu_{2} \frac{\Gamma(39 / 20)}{\Gamma(13)} t^{\frac{3}{10}}+\mu_{2} \frac{\Gamma(19 / 20)}{\Gamma(3 / 10)} t^{-\frac{7}{10}}}{2 \nabla} \int_{0}^{3 / 4} \frac{\left(\frac{3}{4}-s\right)^{\frac{19}{20}}}{\Gamma(39 / 20)} s^{\frac{49}{10}}(1-s)^{-\frac{23}{20}} d s \\
+\frac{\lambda_{2} \frac{\Gamma(39 / 20)}{\Gamma(13 / 10)} t^{\frac{3}{10}}-\omega_{2} \frac{\Gamma(19 / 20)}{\Gamma(3 / 10)} t^{-\frac{7}{10}}}{2 \nabla} \int_{0}^{1 / 2} \frac{\left(\frac{1}{2}-s\right)^{\frac{19}{20}}}{\Gamma(39 / 20)} s^{\frac{49}{10}}(1-s)^{-\frac{23}{20}} d s \mid \\
\leq \frac{\mathbf{B}(3 / 20,59 / 10)}{\Gamma(13 / 10)}+\left(\frac{3}{2} \frac{\nu_{2} \frac{\Gamma(39 / 20)}{\Gamma(13 / 10)}+\mu_{2} \frac{\Gamma(19 / 20)}{\Gamma(3 / 10)}}{\nabla}+\frac{1}{2} \frac{\lambda_{2} \frac{\Gamma(39 / 20)}{\Gamma(13 / 10)}+\omega_{2} \frac{\Gamma(19 / 20)}{\Gamma(3 / 10)}}{\nabla}\right) \frac{\mathbf{B}(4 / 5,59 / 10)}{\Gamma(39 / 20)}
\end{gathered}
$$

$\approx 6.2585$.

It is easy to see by calculation that

$$
\begin{aligned}
& \|\bar{\Phi}\|=\max \left\{\sup _{t \in(0,1]} t^{\frac{1}{10}}|\bar{\Phi}(t)|, \sup _{t \in(0,1]} t^{\frac{9}{10}}\left|D_{0^{+}}^{\frac{4}{5}} \bar{\Phi}(t)\right|\right\} \leq 20.7609 \\
& \|\bar{\Psi}\|=\max \left\{\sup _{t \in(0,1]} t^{\frac{1}{20}}|\bar{\Psi}(t)|, \sup _{t \in(0,1]} t^{\frac{7}{10}}\left|D_{0^{+}}^{\frac{13}{20}} \bar{\Psi}(t)\right|\right\} \leq 6.2585 .
\end{aligned}
$$

One sees that (B1) holds with $A_{i}=B_{i}=C_{i}=D_{i}=0(i=1,2), \Phi_{i 0}(t)=\Psi_{i 0}(t)=0(i=$ $1,2), \Phi_{i}(t)=\Psi_{i}(t)=0(i=1,2), \Phi(t)=t^{2}, \Psi(t)=t^{5}$. 
Furthermore, we have

$$
\begin{aligned}
& M_{1}=\max \left\{b_{1} \frac{\left[\Delta+(1+b)\left(v_{1}+\mu_{1}\right)+a\left(\lambda_{1}+\omega_{1}\right)\right] \mathbf{B}\left(\alpha+l_{1}, k_{1}+1\right)}{\Gamma(\alpha) \Delta},\right. \\
& \frac{b_{1} \mathbf{B}\left(\alpha-m+l_{1}, k_{1}+1\right)}{\left.\Gamma(\alpha-)_{1}\right)} \\
& +a_{1} \frac{\left[v_{1} \frac{\Gamma(\alpha)}{\Gamma(\alpha-m)}+\mu_{1} \frac{\Gamma(\alpha-1)}{|\Gamma(\alpha-m-1)|}+\left(b v_{1} \frac{\Gamma(\alpha)}{\Gamma(\alpha-m)}+b \mu_{1} \frac{\Gamma(\alpha-1)}{\Gamma(\alpha-m-1) \mid}\right) \eta^{\left.\alpha+k_{1}+l_{1}\right]}\right] \mathbf{B}\left(\alpha-m+l_{1}, k_{1}+1\right)}{\Gamma(\alpha) \Delta} \\
& \left.+b_{1} \frac{\left(a \lambda_{1} \frac{\Gamma(\alpha)}{\Gamma(\alpha-m)}+a \omega_{1} \frac{\Gamma(\alpha-1)}{\Gamma(\alpha-m-1) \mid}\right) \xi^{\alpha+k_{1}+l_{1}} \mathbf{B}\left(\alpha-m+l_{1}, k_{1}+1\right)}{\Gamma(\alpha) \Delta}\right\} \\
& \leq b_{1}\left(\frac{\left[\Delta+(1+b)\left(v_{1}+\mu_{1}\right)+a\left(\lambda_{1}+\omega_{1}\right)\right] \mathbf{B}\left(\alpha+l_{1}, k_{1}+1\right)}{\Gamma(\alpha) \Delta}+\frac{\mathbf{B}\left(\alpha-m+l_{1}, k_{1}+1\right)}{\Gamma(\alpha-m)}\right. \\
& \left.+\frac{\left(a \lambda_{1} \frac{\Gamma(\alpha)}{\Gamma(\alpha-m)}+a \omega_{1} \frac{\Gamma(\alpha-1)}{\Gamma(\alpha-m-1) \mid}\right) \xi^{\alpha+k_{1}+l_{1}} \mathbf{B}\left(\alpha-m+l_{1}, k_{1}+1\right)}{\Gamma(\alpha) \Delta}\right) \\
& +a_{1} \frac{\left[v_{1} \frac{\Gamma(\alpha)}{\Gamma(\alpha-m)}+\mu_{1} \frac{\Gamma(\alpha-1)}{|\Gamma(\alpha-m-1)|}+\left(b v_{1} \frac{\Gamma(\alpha)}{\Gamma(\alpha-m)}+b \mu_{1} \frac{\Gamma(\alpha-1)}{\Gamma(\alpha-m-1) \mid}\right) \eta^{\alpha+k_{1}+l_{1}}\right] \mathbf{B}\left(\alpha-m+l_{1}, k_{1}+1\right)}{\Gamma(\alpha) \Delta} \\
& \leq a_{1} \frac{\left[0.0670 \frac{\Gamma(19 / 10)}{\Gamma(11 / 10)}+0.2679 \frac{\Gamma(9 / 10)}{\Gamma(1 / 10)}+\left(\frac{0.0670}{2} \frac{\Gamma(19 / 10)}{\Gamma(11 / 10)}+\frac{0.2679}{2} \frac{\Gamma(9 / 10)}{\Gamma(1 / 10)}\right)\left(\frac{3}{4}\right)^{\frac{3}{4}}\right] \mathrm{B}(1 / 20,9 / 10)}{0.4093 \Gamma(19 / 10)} \\
& +b_{1}\left(\frac{\left[0.4093+\frac{3}{2}(0.0670+0.2679)+\frac{1}{2}(0.4641+0.6141)\right] \mathbf{B}(17 / 20,9 / 10)}{0.4093 \Gamma(19 / 10)}+\frac{\mathbf{B}(1 / 20,9 / 10)}{\Gamma(11 / 10)}\right. \\
& \left.+\frac{\left(\frac{0.4641}{2} \frac{\Gamma(19 / 10)}{\Gamma(11 / 10)}+\frac{0.6141}{2} \frac{\Gamma(9 / 10)}{\Gamma \Gamma(1 / 10) \mid}\right)\left(\frac{1}{2}\right)^{\frac{3}{4}} \mathbf{B}(1 / 20,9 / 10)}{0.4093 \Gamma(19 / 10)}\right) \approx 6.9793 a_{1}+31.0850 b_{1}
\end{aligned}
$$

and

$$
\begin{aligned}
& N_{1}=\max \left\{a_{1} \frac{\left[\Delta+(1+b)\left(v_{1}+\mu_{1}\right)+a\left(\lambda_{1}+\omega_{1}\right)\right] \mathbf{B}\left(\alpha+l_{1}, k_{1}+1\right)}{\Gamma(\alpha) \Delta},\right. \\
& \frac{a_{1} \mathbf{B}\left(\alpha-m+l_{1}, k_{1}+1\right)}{\Gamma(\alpha-m)} \\
& +b_{1} \frac{\left[v_{1} \frac{\Gamma(\alpha)}{\Gamma(\alpha-m)}+\mu_{1} \frac{\Gamma(\alpha-1)}{\Gamma \Gamma(\alpha-m-1)]}+\left(b v_{1} \frac{\Gamma(\alpha)}{\Gamma(\alpha-m)}+b \mu_{1} \frac{\Gamma(\alpha-1)}{\Gamma(\alpha)}\right) \eta^{\left.\alpha+k_{1}+l_{1}\right]}\right] \mathbf{B}\left(\alpha-m+l_{1}, k_{1}+1\right)}{\Gamma(\alpha) \Delta} \\
& \left.+a_{1} \frac{\left(a \lambda_{1} \frac{\Gamma(\alpha)}{\Gamma(\alpha-m)}+a \omega_{1} \frac{\Gamma(\alpha-1)}{\Gamma \Gamma(\alpha-m-1)]}\right) \xi^{\alpha+k_{1}+l_{1} \mathbf{B}\left(\alpha-m+l_{1}, k_{1}+1\right)}}{\Gamma(\alpha) \Delta}\right\} \\
& \leq b_{1} \frac{\left[0.0670 \frac{\Gamma(19 / 10)}{\Gamma(11 / 10)}+0.2679 \frac{\Gamma(9 / 10)}{\Gamma(1 / 10)}+\left(\frac{0.0670}{2} \frac{\Gamma(19 / 10)}{\Gamma(11 / 10)}+\frac{0.2679}{2} \frac{\Gamma(9 / 10)}{\Gamma(1 / 10)}\right)\left(\frac{3}{4}\right)^{\frac{3}{4}}\right] \mathbf{B}(1 / 20,9 / 10)}{0.4093 \Gamma(19 / 10)} \\
& +a_{1}\left(\frac{\left[0.4093+\frac{3}{2}(0.0670+0.2679)+\frac{1}{2}(0.4641+0.6141)\right] \mathbf{B}(17 / 20,9 / 10)}{0.4093 \Gamma(19 / 10)}+\frac{\mathbf{B}(1 / 20,9 / 10)}{\Gamma(11 / 10)}\right. \\
& \left.+\frac{\left(\frac{0.4641}{2} \frac{\Gamma(19 / 10)}{\Gamma(11 / 10)}+\frac{0.6141}{2} \frac{\Gamma(9 / 10)}{\Gamma(1 / 10)]}\right)\left(\frac{1}{2}\right)^{\frac{3}{4}} \mathbf{B}(1 / 20,9 / 10)}{0.4093 \Gamma(19 / 10)}\right) \approx 34.1691 a_{1}+7.0239 b_{1},
\end{aligned}
$$




$$
\begin{aligned}
& M_{2} \leq a_{2} \frac{\left[0.4828 \frac{\Gamma(39 / 20)}{\Gamma(13 / 10)}+0.2588 \frac{\Gamma(19 / 20)}{\Gamma(3 / 10)}+\left(\frac{0.4828}{2} \frac{\Gamma(39 / 20)}{\Gamma(13 / 10)}+\frac{0.2588}{2} \frac{\Gamma(19 / 20)}{\Gamma(3 / 10)}\right)\left(\frac{3}{4}\right)^{\frac{7}{10}}\right] \mathbf{B}(3 / 20,9 / 10)}{0.4237 \Gamma(39 / 20)} \\
& +b_{2}\left(\frac{\left[0.4237+\frac{3}{2}(0.4828+0.2588)+\frac{1}{2}(0.4824+0.6196)\right] \mathbf{B}(4 / 5,9 / 10)}{0.4237 \Gamma(39 / 20)}+\frac{\mathbf{B}(3 / 10,9 / 10)}{0.4237 \Gamma(13 / 10)}\right. \\
& \left.+\frac{\left(\frac{0.4824}{2} \frac{\Gamma(39 / 20)}{\Gamma(13 / / 10)}+\frac{0.6196}{2} \frac{\Gamma(19 / 20)}{\Gamma(3 / 10)}\right)\left(\frac{1}{2}\right)^{\frac{7}{10}} \mathbf{B}(3 / 20,9 / 10)}{0.4237 \Gamma(39 / 20)}\right) \approx 14.2808 a_{2}+19.7870 b_{2},
\end{aligned}
$$

and

$$
\begin{aligned}
& N_{2} \leq b_{2} \frac{\left[0.4828 \frac{\Gamma(39 / 20)}{\Gamma(13 / 10)}+0.2588 \frac{\Gamma(19 / 20)}{\Gamma(3 / 10)}+\left(\frac{0.4828}{2} \frac{\Gamma(39 / 20)}{\Gamma(13 / 10)}+\frac{0.2588}{2} \frac{\Gamma(19 / 20)}{\Gamma(3 / 10)}\right)\left(\frac{3}{4}\right) \frac{7}{10}\right] \mathrm{B}(3 / 20,9 / 10)}{0.4237 \Gamma(39 / 20)} \\
& +a_{2}\left(\frac{\left[0.4237+\frac{3}{2}(0.4828+0.2588)+\frac{1}{2}(0.4824+0.6196)\right] \mathbf{B}(4 / 5,9 / 10)}{0.4237 \Gamma(39 / 20)}+\frac{\mathbf{B}(13 / 20,9 / 10)}{\Gamma(13 / 10)}\right. \\
& \left.+\frac{\left(\frac{0.4828}{2} \frac{\Gamma(39 / 20)}{\Gamma(13 / 10)}+\frac{0.6196}{2} \frac{\Gamma(19 / 20)}{\Gamma(3 / 10)}\right)\left(\frac{1}{2}\right)^{\frac{7}{10}} \mathbf{B}(3 / / 20,9 / 10)}{0.4237 \Gamma(39 / 20)}\right) \approx 12.4878 a_{2}+14.2808 b_{2} .
\end{aligned}
$$

So

$$
\begin{aligned}
& M=M_{1}+N_{1} \leq 41.1484 a_{1}+38.1089 b_{1}, \quad N=M_{2}+N_{2} \leq 26.7686 a_{2}+34.0678 b_{2}, \\
& \Phi_{0}=\max \left\{\|\bar{\Phi}\|_{1}, 1\right\} \leq 20.7609, \quad \Psi_{0}=\max \left\{\|\bar{\Psi}\|_{1}, 1\right\} \leq 6.2585, \\
& \tau=\max \left\{\epsilon_{1}, \delta_{1}\right\}, \quad \sigma=\max \left\{\sigma_{1}, \gamma_{1}\right\} .
\end{aligned}
$$

(i) If there exists a constant $H>0$ such that

$$
\begin{aligned}
& \left|f(t, u, v)-t^{2}\right| \leq H, \quad t \in(0,1), u, v \in \mathbb{R} \\
& \left|g(t, u, v)-t^{5}\right| \leq H, \quad t \in(0,1), u, v \in \mathbb{R}
\end{aligned}
$$

then we can choose $\epsilon_{1}=\delta_{1}=\sigma_{1}=\gamma_{1}=0$. So $\tau \sigma=0<1$. Then (i) in Theorem 3.1 implies that $\operatorname{BVP}(4.1)$ has at least one positive solution.

(ii) If there exist constants $b_{1}, b_{2} \geq 0$ and $\epsilon_{1}, \sigma_{1}>0$ such that

$$
\begin{aligned}
& \left|f(t, u, v)-t^{2}\right| \leq b_{1} t^{\frac{\epsilon_{1}}{20}} u^{\epsilon_{1}}, \\
& \left|g(t, u, v)-t^{5}\right| \leq b_{2} t^{\frac{\sigma_{1}}{10}} u^{\sigma_{1},},
\end{aligned}
$$

we can choose $\delta_{1}=\gamma_{1}=0, a_{1}=a_{2}=0$. Theorem 3.1 implies that $\operatorname{BVP}(4.1)$ has at least one solution if one of the followings holds:

(a) $\epsilon_{1} \sigma_{1}<1$;

(b) $\epsilon_{1} \sigma_{1}=1$ with $\left(38.1089 b_{1}\right)^{1 / \sigma_{1}} 34.0678 b_{2}<1$ or $38.1089 b_{1}\left(34.0678 b_{2}\right)^{1 / \tau_{1}}<1$

(c) $\epsilon_{1} \sigma_{1}>1$ with

$$
\frac{38.1089 b_{1}\left(\epsilon_{1} \sigma_{1}-1\right) \epsilon_{1} \sigma_{1}\left[238.5046 b_{1}+20.7069\right]^{\epsilon_{1} \sigma_{1}-1}}{\left(\epsilon_{1} \sigma_{1}-1\right)^{\epsilon_{1} \sigma_{1}}}\left(34.0678 b_{2}\right)^{\sigma_{1}} \leq 1
$$

or

$$
\frac{34.0678 b_{2}\left(\epsilon_{1} \sigma_{1}-1\right) \epsilon_{1} \sigma_{1}\left[707.2782 b_{2}+6.2585\right]^{\epsilon_{1} \sigma_{1}-1}}{\left(\epsilon_{1} \sigma_{1}-1\right)^{\epsilon_{1} \sigma_{1}}}\left(38.1089 b_{1}\right)^{\epsilon_{1}} \leq 1
$$

(iii) If

$$
\begin{aligned}
& f(t, u, v)=t^{2}+b_{1} t^{\frac{\epsilon_{1}}{20}} u^{\epsilon_{1}}+a_{1} t^{\frac{7 \delta_{1}}{10}} v^{\delta_{1}}, a_{1}, b_{1} \geq 0, \epsilon_{1}, \delta_{1}>0, \\
& g(t, u, v)=t^{5}+b_{2} t^{\frac{\sigma_{1}}{10}} u^{\sigma_{1}}+a_{2} t^{\frac{9 \gamma_{1}}{10}} v^{\gamma_{1}}, a_{2}, b_{2} \geq 0, \sigma_{1}, \gamma_{1}>0,
\end{aligned}
$$


then Theorem 3.1 implies that $\operatorname{BVP}(4.1)$ has at least one positive solution if one of the followings holds:

(a) $\tau \sigma<1$

(b) $\tau \sigma=1$ with $\left.926.7686 a_{2}+34.0678 b_{2}\right)\left(41.1484 a_{1}+38.1089 b_{1}\right)^{1 / \sigma}<1$ or $\left(41.1484 a_{1}+\right.$ $\left.\left.38.1089 b_{1}\right) 926.7686 a_{2}+34.0678 b_{2}\right)^{1 / \tau}<1$

(c) $\tau \sigma>1$ with

$$
\begin{aligned}
& \frac{\left(41.1484 a_{1}+38.1089 b_{1}\right)(\tau \sigma-1) \tau \sigma\left[6.2585\left(41.1484 a_{1}+38.1089 b_{1}\right)+20.7690\right]^{\tau \sigma-1}}{(\tau \sigma-1)^{\tau \sigma}} \\
& \leq \frac{1}{\left.926.7686 a_{2}+34.0678 b_{2}\right)^{\sigma}} \\
& \text { or } \frac{\left(26.7686 a_{2}+34.0678 b_{2}\right)(\tau \sigma-1) \tau \sigma\left[20.7690\left(26.7686 a_{2}+34.0678 b_{2}\right)+6.2585\right]^{\tau \sigma-1}}{(\tau \sigma-1)^{\tau \sigma}} \\
& \leq \frac{1}{\left(41.1484 a_{1}+38.1089 b_{1}\right)^{\tau}} .
\end{aligned}
$$

4.2. Remark. Since both $p$ and $q$ are not measurable on $(0,1)$, we know that all known results in $[1,17]$ can not be applied to establish existence results for solutions of $\mathrm{BVP}(4.1)$. Hence Theorem 3.1 fills a gap not covered by $[1,17]$.

4.3. Example. We consider the following boundary value problem

$$
\left\{\begin{array}{l}
D_{0^{+}}^{\frac{19}{10}} u(t)+t^{-\frac{1}{2}}(1-t)^{-\frac{1}{5}} f\left(t, v(t), D_{0^{+}}^{\frac{39}{40}} v(t)\right)=0, \quad t \in(0,1), \\
D_{0^{+}}^{\frac{39}{20}} v(t)+t^{-\frac{1}{2}}(1-t)^{\frac{1}{10}} g\left(t, u(t), D_{0^{+}}^{\frac{19}{20}} u(t)\right)=0, \quad t \in(0,1), \\
\lim _{t \rightarrow 0} t^{\frac{1}{5}} u(t)-\frac{1}{2} u(1 / 2)=0, \\
u(1)-\frac{1}{2} u(3 / 4)=0, \\
\lim _{t \rightarrow 0} t^{\frac{1}{9}} v(t)-\frac{1}{2} v(1 / 2)=0, \\
v(1)-\frac{1}{2} v(3 / 4)=0,
\end{array}\right.
$$

where

$$
\begin{aligned}
& f(t, u, v)=t^{2}+b_{1} t u^{\epsilon_{1}}+a_{1} t v^{\delta_{1}},{ }_{1}, b_{1} \geq 0, \epsilon_{1}, \delta_{1}>0, \\
& g(t, u, v)=4 t^{5}+b_{2} t u^{\sigma_{1}}+a_{2} t v^{\gamma_{1}}, a_{2}, b_{2} \geq 0, \sigma_{1}, \gamma_{1}>0 .
\end{aligned}
$$

Then $\operatorname{BVP}(4.2)$ has at least one positive solution for sufficiently small $a_{i}, b_{i}(i=1,2)$.

Proof. Corresponding to $\operatorname{BVP}(1.8)$, we have $\alpha=\frac{19}{10}, \beta=\frac{39}{20}, m=\frac{19}{20}$ and $n=\frac{39}{40}$, $a=b=c=d=\frac{1}{2}$ and $\phi_{i}(t, u, v)=\psi_{i}(t, u, v) \equiv 0(i=1,2)$ and $p(t)=t^{-\frac{1}{2}}(1-t)^{-\frac{6}{5}}$, $q(t)=t^{-\frac{1}{2}}(1-t)^{\frac{10}{9}}$.

It is easy to see that (i)-(iv) hold with $k_{1}=-\frac{1}{10}=k_{2}$, and $l_{1}=-\frac{1}{5}, l_{2}=-\frac{1}{10}$. One sees that $k_{1}>-1, \alpha-m+l_{1}>0,2+k_{1}+l_{1}>0, k_{2}>-1, \beta-n+l_{2}>0,2+k_{2}+l_{2}>0$. One sees $m>\alpha-1, n>\beta-1$.

Then Theorem 3.1 implies that BVP(4.2) has at least one positive solution if one of the followings is satisfied:

(I) $\tau \sigma<1$

(II) $\tau \sigma \geq 1$ for sufficiently small $a_{i}, b_{i}(i=1,2)$.

\section{Acknowledgments}

The author would like to thank the referees and editors for their careful reading and some kindly comments on improving the presentation of this paper. 


\section{References}

[1] Ahmad, A. and Nieto, J.J. Existence results for a coupled system of nonlinear fractional differential equations with three-point boundary conditions, Comput. Math. Appl. 58, 18381843, 2009.

[2] Ahmad, B. and Sivasundaram, S. On four-point nonlocal boundary value problems of nonlinear integro-differential equations of fractional order, Appl. Math. Comput. 217, 480-487, 2010.

[3] Avery R.I., and Peterson, A.C. Three Positive Fixed Points of Nonlinear Operators on Ordered Banach Spaces, Comput. Math. Appl. 42, 313-322, 2001.

[4] Basset, A.B. On the descent of a sphere in a vicous liquid, Q. J. Pure Appl. Math. 41, 369-381, 1910.

[5] Bai, C. and Fang, J. The existence of a positive solution for a singular coupled system of nonlinear fractional differential equations, Appl. Math. Comput. 150, (3), 611-621, 2004.

[6] Caponetto, R., Dongona, G. and Fortuna, L. Fractional Differential Systems, Modeling and control applications, World Scientific Series on Nonlinear Science, Ser. A, 72, World Scientific Publishing Co. Pte. Ltd. 2010.

[7] Duan, J. and Temuer, C. Solution for system of linear fractional differential equations with constant coefficients, Journal of Mathematics, 29, 599-603, 2009.

[8] Gaber, M. and Brikaa, M.G. Existence results for a coupled system of nonlinear fractional differential equation with four-point boundary conditions, ISRN Mathematical Analysis, 2011, Article ID 468346, 14 pages, 2011.

[9] Goodrich, C.S. Existence of a positive solution to systems of differential equations of fractional order, Comput. Math. Appl. 62, 1251-1268, 2011.

[10] Liu, B. Positive solutions of a nonlinear four-point boundary value problems in Banach spaces, J. Math. Anal. Appl. 305, 253-276, 2005.

[11] Liu, L., Zhang, X. and Wu, Y. On existence of positive solutions of a two-point boundary value problem for a nonlinear singular semipositone system, Appl. Math. Comput. 192, 223-232, 2007.

[12] Mainardi, F. Fraction Calculus: Some basic problems in continuum and statistical machanics, In: A. Carpinteri, F. Mainardi (eds.) Fratals and Fractional Calculus in Continuum Machanics, (Springer, Vien, 1997), 291-348.

[13] Miller, K.S. and Ross, B. An Introduction to the Fractional Calculus and Fractional Differential Equation, (Wiley, New York, 1993.)

[14] Mamchuev, M.O. Boundary value problem for a system of fractional partial differential equations, Partial Differential Equations, 44, 1737-1749, 2008.

[15] Rehman, M. and Khan, R. A note on boundaryvalueproblems for a coupled system of fractionaldifferential equations, Comput. Math. Appl. 61, 2630-2637, 2011.

[16] Wang, J., Xiang, H. and Liu, Z. Positive Solution to Nonzero Boundary Values Problem for a Coupled System of Nonlinear Fractional Differential Equations, International Journal of Differential Equations, 2010, Article ID 186928, 12 pages, 2010.

[17] $\mathrm{Su}, \mathrm{X}$. Boundary value problem for a coupled system of nonlinear fractional differential equations, Applied Mathematics Letters 22 (1), 64-69, 2009.

[18] Torvik, P.J., and Bagley, R.L. On the appearance of the fractional derivative in the behavior of real materials, J. Appl. Mech. 51, 294-298, 1984.

[19] Trujillo, J.J., Rivero, M. and Bonilla, B. On a Riemann-Liouville generalized Taylor's formula, J. Math. Anal. Appl. 231, 255-265, 1999.

[20] Yuan, C. Multiple positive solutions for $(n-1,1)$-type semipositone conjugate boundary value problems for coupled systems of nonlinear fractional differential equations, E. J. Qualitative Theory of Diff. Equ. 13 1-12, 2012.

[21] Yang, A. and Ge, W. Positive solutions for boundary value problems of N-dimension nonlinear fractional differential systems, Boundary Value Problems 2008, article ID 437453, 2008.

[22] Yuan, C., Jiang, D., O'regan, D. and Agarwal, R.P. Existence and uniqueness of positive solutions of boundary value problems for coupled systems of singular second-order threepoint non-linear differential and difference equations, Appl. Anal. 87 921-932, 2008. 
[23] Yuan, C., Jiang, D., O'regan, D. and Agarwal, R.P. Multiple positive solutions to systems of nonlinear semipositone fractional differential equations with coupled boundary conditions, E. J. Qualitative Theory of Diff. Equ. 13 (2012), 1-17, 2012.

[24] Zou, Y., Liu, L. and Cui, Y. The existence of solutions for four-point coupled boundary value problems of fractional differential equations at resonance, Abstract and Applied Analysis 2014, Article ID 314083, 8 pages, 2014.

[25] Ji, D. and Ge, W. Positive solution for four-point nonlocal boundary value problems of fractional order, Mathematical Methods in the Applied Sciences 37 (8), 1232-1239, 2014.

[26] Zhao, J., Geng, F. and Ge, W. Positive solutions to a new kind Sturm-Liouville-like fourpoint boundary value problem, Applied Mathematics and Computation 217 (2), 811-819, 2010.

[27] Zhao, X., Chai, C. and Ge, W. Positive solutions for fractional four-point boundary value problems, Comm. Nonl. Sci. Numer. Simul. 16 (9), 3665-3672, 2011. 